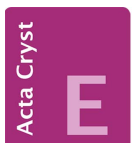

CRYSTALLOGRAPHIC COMMUNICATIONS

ISSN 2056-9890

Received 28 April 2015

Accepted 15 May 2015

Edited by I. D. Brown, McMaster University, Canada

Keywords: crystal structure; usovite; Fecontaining usovite; disorder; fluorides

CCDC reference: 1401194

Supporting information: this article has supporting information at journals.iucr.org/e

\section{Crystal structure of the Fe-member of usovite}

\author{
Matthias Weil
}

Institute for Chemical Technologies and Analytics, Division of Structural Chemistry, Vienna University of Technology, Getreidemarkt 9/164-SC, A-1060 Vienna, Austria. *Correspondence e-mail: mweil@mail.zserv.tuwien.ac.at

Crystals of the title compound, with the idealized composition $\mathrm{Ba}_{2} \mathrm{CaFeAl}_{2} \mathrm{~F}_{14}$, dibarium calcium iron(II) dialuminium tetradecafluoride, were obtained serendipitously by reacting a mixture of the binary fluorides $\mathrm{BaF}_{2}, \mathrm{CaF}_{2}$ and $\mathrm{AlF}_{3}$ in a leaky steel reactor. The compound crystallizes in the usovite structure type $\left(\mathrm{Ba}_{2} \mathrm{CaMgAl}_{2} \mathrm{~F}_{14}\right)$, with $\mathrm{Fe}^{2+}$ cations replacing the $\mathrm{Mg}^{2+}$ cations. The principal building units are distorted $\left[\mathrm{CaF}_{8}\right]$ square-antiprisms (point group symmetry 2 ), $\left[\mathrm{FeF}_{6}\right]$ octahedra (point group symmetry $\overline{1}$ ) and $\left[\mathrm{AlF}_{6}\right]$ octahedra that are condensed into undulating ${ }_{\infty}^{2}\left[\mathrm{CaFeAl}_{2} \mathrm{~F}_{14}\right]^{4-}$ layers parallel (100). The $\mathrm{Ba}^{2+}$ cations separate the layers and exhibit a coordination number of 12 . Two crystal structure models with a different treatment of the disordered Fe site [mixed Fe/Ca occupation, model (I), versus underoccupation of Fe, model (II)], are discussed, leading to different refined formulae $\mathrm{Ba}_{2} \mathrm{Ca}_{1.310 \text { (15)- }}$ $\mathrm{Fe}_{0.690(15)} \mathrm{Al}_{2} \mathrm{~F}_{14}$ [model (I)] and $\mathrm{Ba}_{2} \mathrm{CaFe}_{0.90(1)} \mathrm{Al}_{2} \mathrm{~F}_{14}$ [model (II)].

\section{Chemical context}

Fluoridoaluminates with alkaline earth cations exhibit a rich crystal chemistry (Babel \& Tressaud, 1985; Weil et al., 2001). They are suitable host materials for optical applications as has been shown by luminescence exitation studies of $\mathrm{SrAlF}_{5}$ or $\mathrm{CaAlF}_{5}$ doped with $\mathrm{Pr}^{3+}$ and $\mathrm{Mn}^{2+}$ (van der Kolk et al., 2004). In order to prepare large single crystals of a related fluoridoaluminate with composition $\mathrm{BaCaAlF}_{7}$, a different preparation route was chosen in comparison with the reported crystal-growth procedure. Instead of using a $\mathrm{ZnCl}_{2}$ melt (Werner \& Weil, 2003), a carbon tool steel container shielded

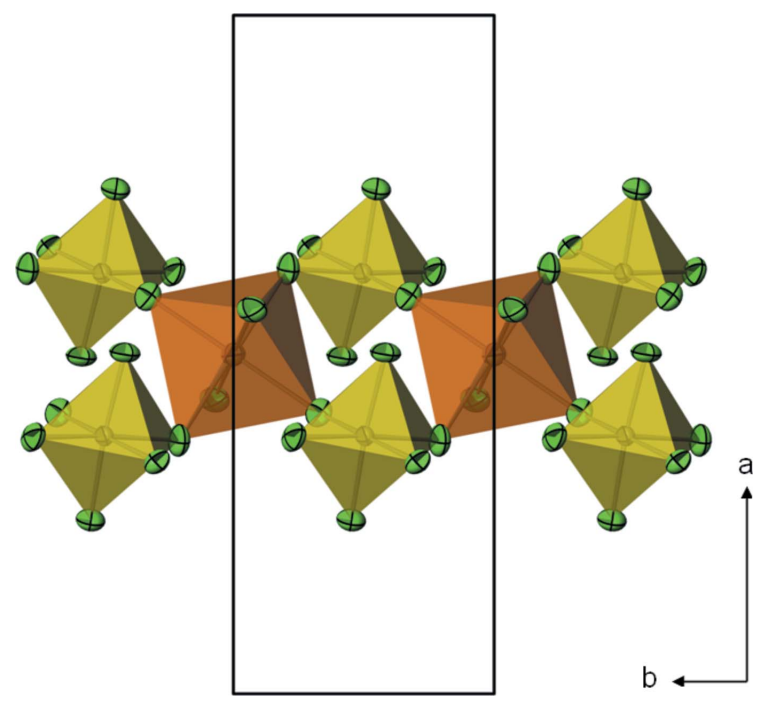

Figure 1

$\left[\mathrm{AlF}_{6}\right]$ octahedra (yellow, with $\mathrm{F}$ atoms green) and $\left[\mathrm{FeF}_{6}\right]$ octahedra (orange) are linked into crosslinked double chains parallel to [010]. Displacement ellipsoids are drawn at the $74 \%$ probability level. 
Table 1

Selected bond lengths ( $)$ for model (I).

For model (II), bond lengths are the same within their standard uncertainties.

\begin{tabular}{|c|c|c|c|}
\hline $\mathrm{Ba}-\mathrm{F} 7$ & $2.696(2)$ & $\mathrm{Ca} 2-\mathrm{F} 4$ & $2.376(2)$ \\
\hline $\mathrm{Ba}-\mathrm{F} 4^{\mathrm{i}}$ & $2.730(2)$ & $\mathrm{Ca} 2-\mathrm{F}^{\mathrm{ix}}$ & $2.376(2)$ \\
\hline $\mathrm{Ba}-\mathrm{F} 1^{\mathrm{i}}$ & $2.755(2)$ & $\mathrm{Ca} 2-\mathrm{F}^{\mathrm{x}}$ & $2.544(2)$ \\
\hline $\mathrm{Ba}-\mathrm{F}^{\mathrm{ii}}$ & $2.765(2)$ & $\mathrm{Ca} 2-\mathrm{F}^{\mathrm{iv}}$ & $2.544(2)$ \\
\hline $\mathrm{Ba}-\mathrm{F}^{\mathrm{iii}}$ & $2.766(2)$ & $\mathrm{Fe} 1-\mathrm{F}^{\mathrm{x}}$ & $2.015(2)$ \\
\hline $\mathrm{Ba}-\mathrm{F}^{5^{\mathrm{iv}}}$ & $2.827(2)$ & $\mathrm{Fe} 1-\mathrm{F}^{\mathrm{i}}$ & $2.015(2)$ \\
\hline $\mathrm{Ba}-\mathrm{F}^{\mathrm{iv}}$ & $2.889(2)$ & $\mathrm{Fe} 1-\mathrm{F}^{\mathrm{xi}}$ & $2.131(2)$ \\
\hline $\mathrm{Ba}-\mathrm{F} 1^{\mathrm{v}}$ & 2.889 (2) & $\mathrm{Fe} 1-\mathrm{F} 2^{\text {viii }}$ & $2.131(2)$ \\
\hline $\mathrm{Ba}-\mathrm{F} 3$ & $2.974(2)$ & $\mathrm{Fe} 1-\mathrm{F}^{\mathrm{x}}$ & $2.216(2)$ \\
\hline $\mathrm{Ba}-\mathrm{F}^{\mathrm{iii}}$ & 3.101 (2) & $\mathrm{Fe} 1-\mathrm{F}^{\mathrm{i}}$ & $2.216(2)$ \\
\hline $\mathrm{Ba}-\mathrm{F}^{\mathrm{iv}}$ & $3.158(2)$ & $\mathrm{Al}-\mathrm{F} 4^{\mathrm{xii}}$ & $1.780(2)$ \\
\hline $\mathrm{Ba}-\mathrm{F} 1^{\mathrm{vi}}$ & $3.233(3)$ & $\mathrm{Al}-\mathrm{F} 1$ & $1.780(3)$ \\
\hline $\mathrm{Ca} 2-\mathrm{F} 7^{\mathrm{vii}}$ & $2.235(2)$ & $\mathrm{Al}-\mathrm{F}^{\mathrm{iv}}$ & $1.790(2)$ \\
\hline $\mathrm{Ca} 2-\mathrm{F}^{\mathrm{i}}$ & $2.235(2)$ & $\mathrm{Al}-\mathrm{F}^{\mathrm{iv}}$ & $1.799(2)$ \\
\hline $\mathrm{Ca} 2-\mathrm{F}^{\mathrm{iii}}$ & $2.369(2)$ & $\mathrm{Al}-\mathrm{F} 5^{\mathrm{iii}}$ & $1.843(2)$ \\
\hline $\mathrm{Ca} 2-\mathrm{F}^{\mathrm{viii}}$ & $2.369(2)$ & $\mathrm{Al}-\mathrm{F} 3^{\mathrm{iii}}$ & $1.846(2)$ \\
\hline
\end{tabular}

Symmetry codes: (i) $-x+\frac{1}{2}, y+\frac{1}{2},-z+\frac{1}{2}$; (ii) $-x+\frac{1}{2}, y+\frac{1}{2},-z+\frac{3}{2}$; (iii) $x,-y+1, z-\frac{1}{2}$; (iv) $-x+\frac{1}{2},-y+\frac{1}{2},-z+1$; (v) $-x+\frac{1}{2}, y-\frac{1}{2},-z+\frac{1}{2}$; (vi) $x,-y+1, z+\frac{1}{2}$; (vii)

with a molybdenum foil was used for solid state reactions between a mixture of the binary fluorides (Weil \& Kubel, 2002). However, during one of these experiments it turned out that the container was not completely lined by the molybdenum foil which consequently led to a reaction with the container wall and an incorporation of iron into parts of the reaction products. Crystal structure analysis of selected crystals from this reaction batch revealed an Fe-containing phase that crystallizes isotypically with the mineral usovite, $\mathrm{Ba}_{2} \mathrm{CaMgAl}_{2} \mathrm{~F}_{14}$ (Litvin et al., 1980).

Compounds with the usovite-type structure are represented by the general formula $\mathrm{Ba}_{2}\left(M^{\mathrm{II}} 1\right)\left(M^{\mathrm{II}} 2\right)\left(M^{\mathrm{III}} 3\right)_{2} \mathrm{~F}_{14}\left(M^{\mathrm{II}} 1=\right.$ $\mathrm{Ca}, \mathrm{Cd}, \mathrm{Mn} ; M^{\mathrm{II}} 2=\mathrm{Mg}, \mathrm{Co}, \mathrm{Mn}, \mathrm{Cu}, \mathrm{Cd}, \mathrm{Fe} ; M^{\mathrm{III}} 3=\mathrm{Al}, \mathrm{V}, \mathrm{Fe}$, $\mathrm{Cr}, \mathrm{Ga}, \mathrm{Mn}$ ) and crystallize with four formula units in the space group $C 2 / c$. Most of the usovite-type representatives known so far were prepared and structurally determined by Babel, Tressaud and co-workers over the last three decades (Holler et al., 1984, 1985; Kaiser et al., 2002; Le Lirzin et al., 1990, 1991, 2008; Qiang et al., 1988).

\section{Structural commentary}

The principal building units of the usovite crystal structure are distorted $\left[\mathrm{BaF}_{12}\right]$ polyhedra, $\left[\left(M^{\mathrm{II}} 1\right) \mathrm{F}_{8}\right]$ sqare-antiprisms (point group symmetry 2 ) and $\left[\left(M^{\mathrm{II}} 2\right) \mathrm{F}_{6}\right]$ octahedra (point group symmetry $\overline{1})$, as well as rather regular $\left[\left(M^{\mathrm{III}} 3\right) \mathrm{F}_{6}\right]$ octahedra. The $\left[\left(M^{\mathrm{II}} 2\right) \mathrm{F}_{6}\right]$ and $\left[\left(M^{\mathrm{III}} 3\right) \mathrm{F}_{6}\right]$ octahedra are connected by corner-sharing into infinite crosslinked double chains ${ }_{\infty}^{1}\left[\left(M^{\mathrm{II}} 2\right) \mathrm{F}_{2} \mathrm{~F}_{4 / 2}\left(M^{\mathrm{III}} 3\right)_{2} \mathrm{~F}_{8} \mathrm{~F}_{4 / 2}\right]$ extending parallel to [010] (Fig. 1). Neighbouring chains are linked by the $\left[\left(M^{\mathrm{I}} 1\right) \mathrm{F}_{8}\right]$ square-antiprisms into undulating (100) layers with composition $\left.{ }_{\infty}^{2}\left[\left(M^{\mathrm{II}} 1\right)\left(M^{\mathrm{II}} 2\right)\left(M^{\mathrm{III}} 3\right)_{2} \mathrm{~F}_{14}\right)\right]^{4-}$, with the $\mathrm{Ba}^{2+}$ cations separating the individual layers (Fig. 2).

The unit-cell volume of the title compound $\left[1067.9\right.$ (2) $\left.\AA^{3}\right]$ is slightly larger than that of usovite $\mathrm{Ba}_{2} \mathrm{CaMgAl}_{2} \mathrm{~F}_{14}$ (1027.9 А; Litvin et al., 1980) due to the replacement of the $\mathrm{Mg}^{2+}$ cations (ionic radius $=0.72 \AA$; Shannon, 1976) by the

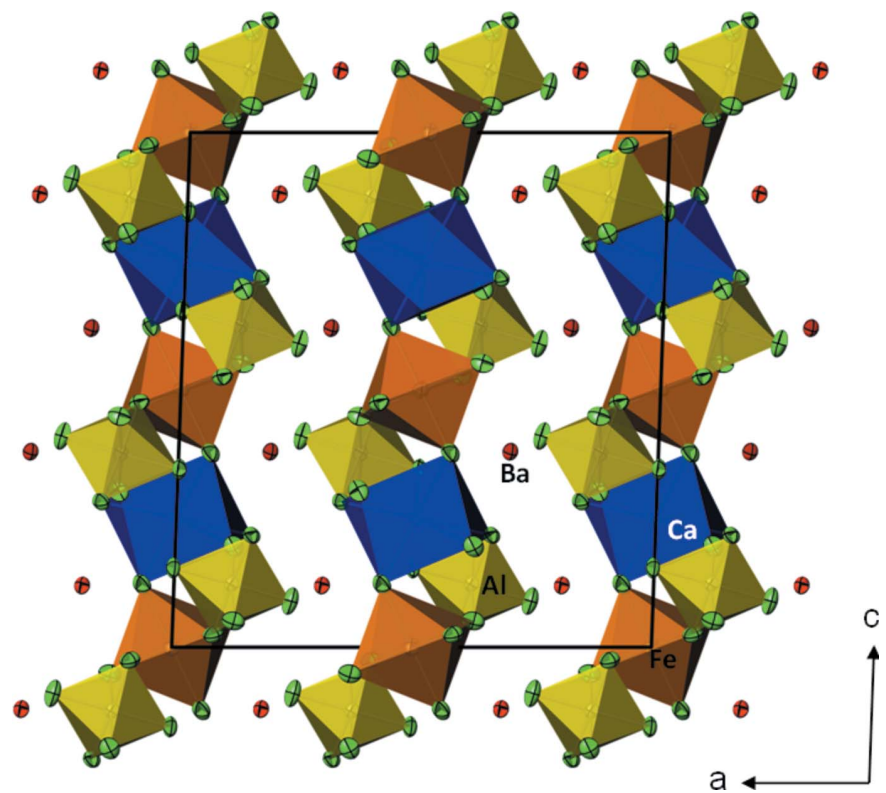

Figure 2

The crystal structure of the usovite-type title compound, emphasizing the formation of the layered ${ }^{2}\left[\mathrm{CaFeAl}_{2} \mathrm{~F}_{14}\right]^{4-}$ framework parallel to (100), separated by $\mathrm{Ba}^{2+}$ cations. Displacement ellipsoids are drawn at the $74 \%$ probability level. The colour code is as in Fig. 1 , with $\left[\mathrm{CaF}_{8}\right]$ polyhedra in blue and $\mathrm{Ba}$ atoms in red.

larger $\mathrm{Fe}^{2+}$ cations (ionic radius $=0.78 \AA$; Shannon, 1976) at the $M^{\mathrm{II}} 2$ site. This is also reflected by the bond lengths within the individual coordination polyhedra (Table 1). Wheras the $\mathrm{Ba}-\mathrm{F}, \mathrm{Ca}-\mathrm{F}$ and $\mathrm{Al}-\mathrm{F}$ distances remain nearly unaltered between the two structures, the $\mathrm{Mg}-\mathrm{F}$ and $\mathrm{Fe}-\mathrm{F}$ distances show remarkable differences. The $\mathrm{Mg}-\mathrm{F}$ distances in the usovite structure range from 1.939 to $2.041 \AA$, the corresponding $\mathrm{Fe}-\mathrm{F}$ distances in the title structure from 2.015 (2) to 2.216 (2) $\AA$, with a mean distance of $2.123 \AA$. The latter is in reasonable agreement with the mean $\mathrm{Fe}^{\mathrm{II}}-\mathrm{F}$ distance of $2.106 \AA$ in the isotypic crystal structure of $\mathrm{Ba}_{2} \mathrm{CaFeV}_{2} \mathrm{~F}_{14}$ (Kaiser et al., 2002). However, the mean bond lengths in both the title structure and $\mathrm{Ba}_{2} \mathrm{CaFeV}_{2} \mathrm{~F}_{14}$ are considerably longer than that of $2.074 \AA$ in the structure of the binary compound $\mathrm{FeF}_{2}$ (Jauch et al., 1993).

A similar increase of the $M-\mathrm{F}$ bond lengths of the $\left[\left(M^{\mathrm{II}} 2\right) \mathrm{F}_{6}\right]$ octahedra was also observed for a series of other usovite-type structures and was associated with an occupational disorder of the $M^{\mathrm{II}} 2$ site. For these models, either a mutual substitution of $\mathrm{Ca}^{2+}$ (on the $M^{\mathrm{II}} 1$ site) with corresponding divalent transition metal ions on the $M^{\mathrm{II}} 2$ site, or partial replacement of the divalent transition metal ions by $\mathrm{Ca}^{2+}$ at the $M^{\mathrm{II}} 2$ site was considered, resulting in stoichiometric compounds and Ca-richer compounds, respectively (Kaiser et al., 2002). In the case of the title compound, a model with mutual substitution of $\mathrm{Ca}^{2+}$ and $\mathrm{Fe}^{2+}$ on the $M^{\mathrm{II}} 1$ and $M^{\mathrm{II}} 2$ sites could be ruled out during refinement. However, a model with an incorporation of $\mathrm{Ca}^{2+}$ on the $\mathrm{Fe}^{2+}$ site resulted in a ratio of $\mathrm{Ca}: \mathrm{Fe}=0.155(7): 0.345$ (7) for this site [model (I); overall refined formula for the compound: $\mathrm{Ba}_{2} \mathrm{Ca}_{1.310(15)^{-}}$ $\left.\mathrm{Fe}_{0.690(15)} \mathrm{Al}_{2} \mathrm{~F}_{14}\right]$ and converged with the same reliability 
Table 2

Bond-valence sum calculations for model (I) in valence units.

\begin{tabular}{lllll}
\hline Atom & $\begin{array}{l}\text { Assumed } \\
\text { valence } \\
\text { state }\end{array}$ & $\begin{array}{l}\text { Bond-valence } \\
\text { sum }\end{array}$ & $\begin{array}{l}\text { Deviation from } \\
\text { assumed valence } \\
\text { state in valence } \\
\text { units }\end{array}$ & $\begin{array}{l}\text { Bond-valence sum } \\
\text { under consideration } \\
\text { of mixed Fe1:Ca1 } \\
\text { occupancy* }\end{array}$ \\
\hline Ba & 2 & 1.94 & 0.06 & 1.94 \\
Fe1 & 2 & 1.73 & 0.27 & 2.00 \\
Ca2 & 2 & 1.95 & 0.05 & 1.95 \\
Al & 3 & 2.97 & 0.03 & 2.97 \\
F1 & 1 & 0.96 & 0.04 & 0.96 \\
F2 & 1 & 0.99 & 0.01 & 1.03 \\
F3 & 1 & 0.93 & 0.07 & 0.96 \\
F4 & 1 & 1.00 & 0 & 1.00 \\
F5 & 1 & 0.99 & 0.01 & 0.99 \\
F6 & 1 & 0.92 & 0.08 & 0.92 \\
F7 & 1 & 0.97 & 0.03 & 1.03 \\
\hline
\end{tabular}

Note: $(*)$ calculated with the weighted average Fe:Ca ratio of 0.77:0.23 at the $M 2$ site.

factors and remaining electron densities as the model without an incorporation of $\mathrm{Ca}^{2+}$ and underoccupation of the $\mathrm{Fe}^{2+}$ site only [model (II); Table 3]. The refined formula for this model is $\mathrm{Ba}_{2} \mathrm{Ca}_{2} \mathrm{Fe}_{0.90(1)} \mathrm{Al}_{2} \mathrm{~F}_{14}$. Bond lengths and angles of the two models are the same within the corresponding standard uncertainties (Table 1).

Kaiser et al. (2002) have discussed in detail the pros and cons of the incorporation of $\mathrm{Ca}^{2+}$ (ionic radius $=1.0 \AA$; Shannon, 1976) at the $M^{\mathrm{II}} 2$ site for various usovite-type structures. Strong arguments supporting an $M^{\mathrm{II}} 2$ site with mixed $\mathrm{Fe} / \mathrm{Ca}$ occupation are the resulting bond-valence sums
(Brown, 2002) that deviate significantly from the expected values of 2 if only $\mathrm{Fe}^{2+}$ ions are considered to be present at the $M^{\mathrm{II}} 2$ site (Table 2). Contrariwise, the bond-valence sums are in excellent agreement with the expected value if a mixed $\mathrm{Fe} / \mathrm{Ca}$ occupancy is taken into account. The corresponding numbers are listed in Table 2 and were calculated with the weighted average occupancy ratio of $\mathrm{Fe}: \mathrm{Ca}=0.77: 0.23$ that was estimated by the program VaList (Wills, 2010). This ratio is in good agreement with the occupancy ratio from the refinement [model (I): Fe:Ca $=0.69: 0.31]$. The resulting global instability index (Brown, 2002) of 0.04 valence units for model (I) suggests a very tightly bonded structure with little strain. Any strain inherent in the usovite structure is obviously relieved by the substitution of $\mathrm{Ca}^{2+}$ on the $M^{\mathrm{II}} 2$ site.

On the other hand, an $M^{\mathrm{II}} 2$ site without an incorporation of $\mathrm{Ca}^{2+}$ would result in an underoccupation of $\mathrm{Fe}^{2+}$ [model (II)] and consequently requires the presence of an element in a higher oxidation state (here most probably $\mathrm{Fe}^{3+}$ ) to compensate the negative charge of -2 of the $\left[\mathrm{Ba}_{2} \mathrm{CaAl}_{2} \mathrm{~F}_{14}\right]$ framework. Although in this case rather a decrease of $M^{\mathrm{II}} 2-\mathrm{F}$ bond lengths should be expected (contrary to the findings of the current study), it cannot competely ruled out that $\mathrm{Fe}^{3+}$ ions are present at this site. As a matter of fact, based on diffraction data alone, there is a clear tendency towards model (I) but no definite answer whether $\mathrm{Fe}$ is partly replaced by $\mathrm{Ca}$ on the $M^{\mathrm{II}} 2$ site [model (I)] or is statistically occupied by $\mathrm{Fe}^{2+}$ and small amounts of $\mathrm{Fe}^{3+}$ [model (II)]. Complementary analytical techniques like Mössbauer spectroscopy will be needed in future to shed some light on this problem.

Table 3

Experimental details.

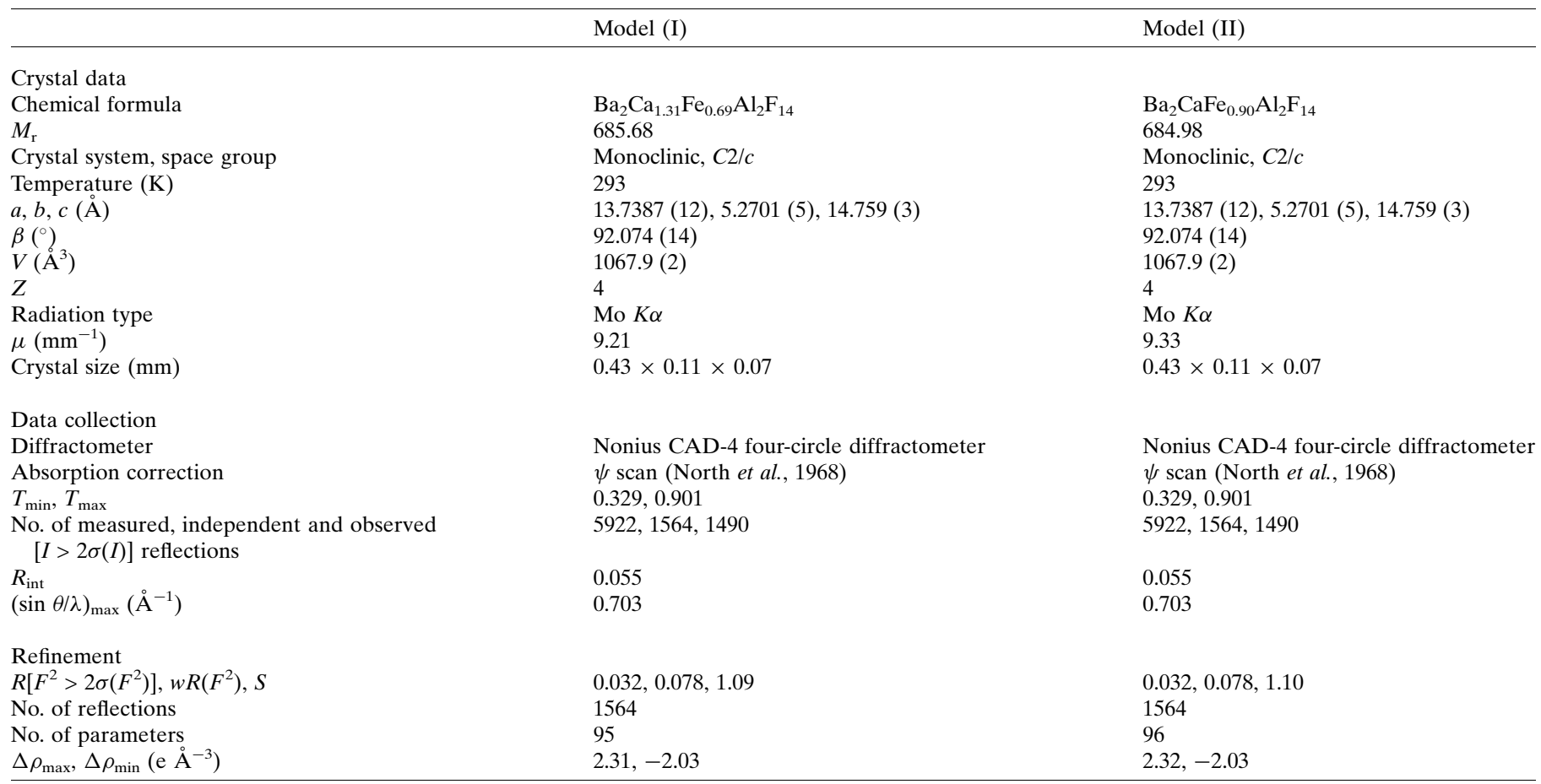

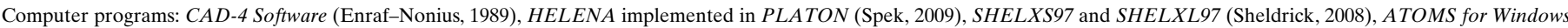
(Dowty, 2006) and publCIF (Westrip, 2010). 


\section{Synthesis and crystallization}

The binary fluorides $\mathrm{AlF}_{3}$ (Merck, Patinal), $\mathrm{CaF}_{2}$ (Merck, Suprapur) and $\mathrm{BaF}_{2}$ (Riedel de Haen, pure) were mixed in the stoichiometric ratio 1:1:1 and thoroughly ground in a ball mill, pressed into tablets and placed in a carbon tool steel container shielded with a molybdenum foil. $\mathrm{NH}_{4} \mathrm{~F} \cdot \mathrm{HF}$ (100 mg, Fluka, p.A.) were added to the mixture to increase the HF pressure, to expel the remaining oxygen and to adjust a slightly reducing atmosphere during the reaction. The reactor was then closed and heated to $1173 \mathrm{~K}$ in the course of $20 \mathrm{~h}$, kept at that temperature for $24 \mathrm{~h}$, and then cooled slowly to $973 \mathrm{~K}$ at a rate of $10 \mathrm{~K} \mathrm{~h}^{-1}$, kept at this temperature for $24 \mathrm{~h}$ and finally cooled to room temperature overnight. After opening the reactor it became evident that parts of the molybdenum foil were torn apart accompanied by a severe attack of the inner container wall. Single crystals of the title compound were separated from the obtained colourless to light-green bulk material. X-ray powder diffraction of the bulk revealed the formation of $\alpha-\mathrm{BaCaAlF}_{7}$ as the main phase and the title compound as a minority phase. Some additional reflections were also present that could not be assigned to any known phases.

\section{Refinement}

Crystal data, data collection and structure refinement details are summarized in Table 3. Coordinates of usovite (Litvin et $a l ., 1980)$ were used as starting parameters for refinement. The model converged rather smoothly with $R 1=0.034$ and $w R 2=$ 0.089 . However, negative residual electron density at the $\mathrm{Fe}$ atom pointed to an underoccupation and/or a statistical disorder of the $M^{\mathrm{II}} 2$ site with a lighter element present. In fact, free refinement of the site occupation factor for this site resulted in only $90 \%$ occupancy and significant better reliability factors (see Table 3 ). The same procedure applied for all other atoms resulted in full occupancy within the twofold standard uncertainty. For the final models, full occupancy was therefore considered for all atoms except Fe. Model (I) accounts for an incorporation of $\mathrm{Ca}^{2+}$ at the $\mathrm{Fe}$ site under consideration of full occupancy; in model (II), the site occupation factor of the Fe site was refined freely without contribution of $\mathrm{Ca}^{2+}$ at this site. The remaining electron densities (Table 3) are virtually the same for both models. They are associated with truncation effects close to the heavy Ba sites, with the maximum electron density $0.68 \AA$ and the minimum electron density $0.96 \AA$ away from the Ba atom.

\section{Acknowledgements}

The X-ray centre of the Vienna University of Technology is acknowledged for providing access to the single-crystal diffractometer. I thank I. D. Brown for very helpful comments and suggestions regarding the bond-valence method.

\section{References}

Babel, D. \& Tressaud, A. (1985). Inorganic Solid Fluorides, edited by P. Hagenmuller, pp. 79-203. Orlando: Academic Press.

Brown, I. D. (2002). In The Chemical Bond in Inorganic Chemistry: The Bond Valence Model. Oxford University Press.

Dowty, E. (2006). ATOMS for Windows. Shape Software, Kingsport, Tennessee, USA.

Enraf-Nonius (1989). CAD-4 Software. Enraf-Nonius, Delft, The Netherlands.

Holler, H., Babel, D., Samouel, M. \& de Kozak, A. (1984). Rev. Chim. Miner. 21, 358-369.

Holler, H., Pebler, J. \& Babel, D. (1985). Z. Anorg. Allg. Chem. 522, 189-201.

Jauch, W., Palmer, A. \& Schultz, A. J. (1993). Acta Cryst. B49, 984-987.

Kaiser, V., Le Lirzin, A., Darriet, J., Tressaud, A., Holler, H. \& Babel, D. (2002). Z. Anorg. Allg. Chem. 628 2617-2626.

Kolk, E. van der, Dorenbos, P., Van Eijk, C. W. E., Vink, A. P., Weil, M. \& Chaminade, J.-P. (2004). J. Appl. Phys. 95, 7867-7872.

Le Lirzin, A., Darriet, J., Tressaud, A. \& Babel, D. (2008). Z. Anorg. Allg. Chem. 634 2737-2739.

Le Lirzin, A., Quiang, X., Darriet, J., Soubeyroux, J. L., Kaiser, V., Pebler, J. \& Babel, D. (1990). Eur. J. Solid State Inorg. Chem. 27, 791-803.

Le Lirzin, A., Soubeyroux, J. L., Tressaud, A., Georges, R. \& Darriet, J. (1991). J. Chim. Phys. Phys.-Chim. Biol. 88, 2173-2189.

Litvin, A. L., Petrunina, A. A., Ostapenko, S. S. \& Povarennykh, A. S. (1980). Dopov. Akad. Nauk Ukr. RSR Ser. pp. 47-50.

North, A. C. T., Phillips, D. C. \& Mathews, F. S. (1968). Acta Cryst. A24, 351-359.

Qiang, X., Darriet, J., Tressaud, A., Soubeyroux, J. L. \& Hagenmuller, P. (1988). Mater. Res. Bull. 23, 637-645.

Shannon, R. D. (1976). Acta Cryst. A32, 751-767.

Sheldrick, G. M. (2008). Acta Cryst. A64, 112-122.

Spek, A. L. (2009). Acta Cryst. D65, 148-155.

Weil, M. \& Kubel, F. (2002). J. Solid State Chem. 164, 150-156.

Weil, M., Zobetz, E., Werner, F. \& Kubel, F. (2001). Solid State Sci. 3, 441-453.

Werner, F. \& Weil, M. (2003). Acta Cryst. E59, i17-i19.

Westrip, S. P. (2010). J. Appl. Cryst. 43, 920-925.

Wills, A. S. (2010). VaList. Program available from www.CCP14.ac. uk. 


\section{supporting information}

Acta Cryst. (2015). E71, 640-643 [doi:10.1107/S2056989015009251]

\section{Crystal structure of the Fe-member of usovite}

\section{Matthias Weil}

\section{Computing details}

Data collection: CAD-4 Software (Enraf-Nonius, 1989) for modelI; CAD-4 Software (Enraf-Nonius, 1989) for modelII. Cell refinement: CAD-4 Software (Enraf-Nonius, 1989) for modelI; CAD-4 Software (Enraf-Nonius, 1989) for modelII. For both compounds, data reduction: HELENA implemented in PLATON (Spek, 2009); program(s) used to solve structure: SHELXS97 (Sheldrick, 2008); program(s) used to refine structure: SHELXL97 (Sheldrick, 2008). Molecular graphics: ATOMS for Windows (Dowty, 2006) for modelI; Atoms for Windows (Dowty, 2006) for modellI. For both compounds, software used to prepare material for publication: publCIF (Westrip, 2010).

(modelI) Dibarium calcium iron(II) dialuminium tetradecafluoride

Crystal data

$\mathrm{Ba}_{2} \mathrm{CaFeAl}_{2} \mathrm{~F}_{14}$

$M_{r}=685.68$

Monoclinic, $C 2 / c$

Hall symbol: $-\mathrm{C} 2 \mathrm{yc}$

$a=13.7387$ (12) $\AA$

$b=5.2701(5) \AA$

$c=14.759(3) \AA$

$\beta=92.074(14)^{\circ}$

$V=1067.9(2) \AA^{3}$

$Z=4$

\section{Data collection}

Nonius CAD-4 four-circle diffractometer

Radiation source: fine-focus sealed tube Graphite monochromator $\omega / 2 \theta$ scans

Absorption correction: $\psi$ scan

(North et al., 1968)

$T_{\min }=0.329, T_{\max }=0.901$

5922 measured reflections

Refinement

Refinement on $F^{2}$

Least-squares matrix: full

$R\left[F^{2}>2 \sigma\left(F^{2}\right)\right]=0.032$

$w R\left(F^{2}\right)=0.078$

$S=1.09$

1564 reflections

95 parameters
$F(000)=1233$

$D_{\mathrm{x}}=4.265 \mathrm{Mg} \mathrm{m}^{-3}$

Mo $K \alpha$ radiation, $\lambda=0.71073 \AA$

Cell parameters from 25 reflections

$\theta=16.0-28.7^{\circ}$

$\mu=9.21 \mathrm{~mm}^{-1}$

$T=293 \mathrm{~K}$

Lath, colourless

$0.43 \times 0.11 \times 0.07 \mathrm{~mm}$

1564 independent reflections

1490 reflections with $I>2 \sigma(I)$

$R_{\text {int }}=0.055$

$\theta_{\text {max }}=30.0^{\circ}, \theta_{\text {min }}=2.8^{\circ}$

$h=-19 \rightarrow 19$

$k=-7 \rightarrow 7$

$l=-20 \rightarrow 20$

3 standard reflections every $240 \mathrm{~min}$ intensity decay: none

0 restraints

Primary atom site location: isomorphous structure methods

$w=1 /\left[\sigma^{2}\left(F_{\mathrm{o}}^{2}\right)+(0.0527 P)^{2}+2.0371 P\right]$

where $P=\left(F_{\mathrm{o}}^{2}+2 F_{\mathrm{c}}{ }^{2}\right) / 3$

$(\Delta / \sigma)_{\max }<0.001$

$\Delta \rho_{\max }=2.31 \mathrm{e} \AA^{-3}$ 
$\Delta \rho_{\min }=-2.03$ e $\AA^{-3}$
Extinction correction: SHELXL97 (Sheldrick, 2008), $\mathrm{Fc}^{*}=\mathrm{kFc}\left[1+0.001 \mathrm{xFc}^{2} \lambda^{3} / \sin (2 \theta)\right]^{-1 / 4}$

Extinction coefficient: $0.0022(2)$

Special details

Geometry. All e.s.d.'s (except the e.s.d. in the dihedral angle between two 1.s. planes) are estimated using the full covariance matrix. The cell e.s.d.'s are taken into account individually in the estimation of e.s.d.'s in distances, angles and torsion angles; correlations between e.s.d.'s in cell parameters are only used when they are defined by crystal symmetry. An approximate (isotropic) treatment of cell e.s.d.'s is used for estimating e.s.d.'s involving 1.s. planes.

Refinement. Refinement of $F^{2}$ against ALL reflections. The weighted $R$-factor $w R$ and goodness of fit $S$ are based on $F^{2}$, conventional $R$-factors $R$ are based on $F$, with $F$ set to zero for negative $F^{2}$. The threshold expression of $F^{2}>\sigma\left(F^{2}\right)$ is used only for calculating $R$-factors(gt) $e t c$. and is not relevant to the choice of reflections for refinement. $R$-factors based on $F^{2}$ are statistically about twice as large as those based on $F$, and $R$ - factors based on ALL data will be even larger.

Fractional atomic coordinates and isotropic or equivalent isotropic displacement parameters $\left(\AA^{2}\right)$

\begin{tabular}{llllll}
\hline & $x$ & $y$ & $z$ & $U_{\text {iso }} * / U_{\text {eq }}$ & Occ. $(<1)$ \\
\hline Ba & $0.308721(14)$ & $0.46574(4)$ & $0.379963(13)$ & $0.01408(12)$ & \\
Fe1 & 0.0000 & 0.5000 & 0.0000 & $0.0122(2)$ & $0.690(15)$ \\
Ca1 & 0.0000 & 0.5000 & 0.0000 & $0.0122(2)$ & $0.310(15)$ \\
Ca2 & 0.0000 & $0.44616(15)$ & 0.2500 & $0.01239(19)$ & \\
Al & $0.38007(8)$ & $0.50390(18)$ & $0.12342(7)$ & $0.0111(2)$ & \\
F1 & $0.25596(18)$ & $0.4521(4)$ & $0.0896(2)$ & $0.0240(5)$ & \\
F2 & $0.08574(15)$ & $0.1721(4)$ & $0.97491(13)$ & $0.0184(4)$ & \\
F3 & $0.37359(16)$ & $0.2100(4)$ & $0.55132(14)$ & $0.0197(4)$ & \\
F4 & $0.00268(16)$ & $0.0837(4)$ & $0.15434(15)$ & $0.0190(4)$ & \\
F5 & $0.34180(14)$ & $0.2933(4)$ & $0.71743(13)$ & $0.0157(4)$ & \\
F6 & $0.12186(16)$ & $0.2677(4)$ & $0.80391(15)$ & $0.0207(4)$ & \\
F7 & $0.43370(17)$ & $0.0715(4)$ & $0.37866(16)$ & $0.0208(4)$ & \\
\end{tabular}

Atomic displacement parameters $\left(\AA^{2}\right)$

\begin{tabular}{lllllll}
\hline & $U^{11}$ & $U^{22}$ & $U^{33}$ & $U^{12}$ & $U^{13}$ & $U^{23}$ \\
\hline Ba & $0.01226(15)$ & $0.01401(16)$ & $0.01593(15)$ & $-0.00069(5)$ & $-0.00023(9)$ & $0.00074(5)$ \\
Fe1 & $0.0106(4)$ & $0.0124(3)$ & $0.0136(4)$ & $0.0004(2)$ & $-0.0005(3)$ & $-0.0029(2)$ \\
Ca1 & $0.0106(4)$ & $0.0124(3)$ & $0.0136(4)$ & $0.0004(2)$ & $-0.0005(3)$ & $-0.0029(2)$ \\
Ca2 & $0.0131(4)$ & $0.0124(4)$ & $0.0118(4)$ & 0.000 & $0.0019(3)$ & 0.000 \\
A1 & $0.0114(5)$ & $0.0096(3)$ & $0.0123(5)$ & $-0.0007(3)$ & $-0.0004(4)$ & $0.0005(3)$ \\
F1 & $0.0130(11)$ & $0.0219(11)$ & $0.0366(14)$ & $-0.0017(8)$ & $-0.0069(10)$ & $-0.0007(9)$ \\
F2 & $0.0218(10)$ & $0.0183(9)$ & $0.0148(8)$ & $0.0033(8)$ & $-0.0016(7)$ & $-0.0026(7)$ \\
F3 & $0.0269(11)$ & $0.0140(8)$ & $0.0184(9)$ & $-0.0003(8)$ & $0.0027(8)$ & $-0.0032(7)$ \\
F4 & $0.0100(9)$ & $0.0247(9)$ & $0.0224(10)$ & $-0.0014(8)$ & $0.0000(7)$ & $-0.0087(8)$ \\
F5 & $0.0155(9)$ & $0.0173(8)$ & $0.0145(8)$ & $-0.0037(7)$ & $0.0027(6)$ & $0.0004(7)$ \\
F6 & $0.0220(10)$ & $0.0161(8)$ & $0.0243(10)$ & $0.0037(8)$ & $0.0035(8)$ & $0.0076(8)$ \\
F7 & $0.0183(11)$ & $0.0239(10)$ & $0.0206(10)$ & $0.0009(9)$ & $0.0052(8)$ & $-0.0067(8)$ \\
\hline
\end{tabular}

Geometric parameters $\left(\AA,{ }^{\circ}\right)$

\begin{tabular}{llll}
\hline $\mathrm{Ba}-\mathrm{F} 7$ & $2.696(2)$ & $\mathrm{Ca} 2-\mathrm{F} 4$ & $2.376(2)$ \\
$\mathrm{Ba}-\mathrm{F} 4^{\mathrm{i}}$ & $2.730(2)$ & $\mathrm{Ca} 2-\mathrm{F}^{\text {ix }}$ & $2.376(2)$
\end{tabular}




\begin{tabular}{|c|c|c|c|}
\hline $\mathrm{Ba}-\mathrm{F} 1^{\mathrm{i}}$ & $2.755(2)$ & $\mathrm{Ca} 2-\mathrm{F}^{\mathrm{x}}$ & $2.544(2)$ \\
\hline $\mathrm{Ba}-\mathrm{F}^{\mathrm{ii}}$ & $2.765(2)$ & $\mathrm{Ca} 2-\mathrm{F}^{\mathrm{iv}}$ & $2.544(2)$ \\
\hline $\mathrm{Ba}-\mathrm{F} 5^{\mathrm{iii}}$ & $2.766(2)$ & $\mathrm{Fe} 1-\mathrm{F}^{\mathrm{x}}$ & $2.015(2)$ \\
\hline $\mathrm{Ba}-\mathrm{F}^{\text {iv }}$ & $2.827(2)$ & $\mathrm{Fe} 1-\mathrm{F}^{\mathrm{i}}$ & $2.015(2)$ \\
\hline $\mathrm{Ba}-\mathrm{F} 3^{\mathrm{iv}}$ & $2.889(2)$ & $\mathrm{Fe} 1-\mathrm{F} 2^{\mathrm{xi}}$ & $2.131(2)$ \\
\hline $\mathrm{Ba}-\mathrm{F} 1^{\mathrm{v}}$ & $2.889(2)$ & $\mathrm{Fe} 1-\mathrm{F} 2^{\text {viii }}$ & $2.131(2)$ \\
\hline $\mathrm{Ba}-\mathrm{F} 3$ & $2.974(2)$ & $\mathrm{Fe} 1-\mathrm{F}^{\mathrm{x}}$ & $2.216(2)$ \\
\hline $\mathrm{Ba}-\mathrm{F} 6^{\mathrm{iii}}$ & $3.101(2)$ & $\mathrm{Fe} 1-\mathrm{F} 3^{\mathrm{i}}$ & $2.216(2)$ \\
\hline $\mathrm{Ba}-\mathrm{F}^{\mathrm{iv}}$ & $3.158(2)$ & $\mathrm{Al}-\mathrm{F} 4^{\mathrm{xii}}$ & $1.780(2)$ \\
\hline $\mathrm{Ba}-\mathrm{F} 1^{\mathrm{vi}}$ & $3.233(3)$ & $\mathrm{Al}-\mathrm{F} 1$ & $1.780(3)$ \\
\hline $\mathrm{Ca} 2-\mathrm{F}^{\mathrm{vii}}$ & $2.235(2)$ & $\mathrm{Al}-\mathrm{F}^{\mathrm{iv}}$ & $1.790(2)$ \\
\hline $\mathrm{Ca} 2-\mathrm{F}^{\mathrm{i}}$ & $2.235(2)$ & $\mathrm{Al}-\mathrm{F} 2^{\mathrm{iv}}$ & $1.799(2)$ \\
\hline $\mathrm{Ca} 2-\mathrm{F} 6^{\mathrm{iii}}$ & $2.369(2)$ & $\mathrm{Al}-\mathrm{F}^{\mathrm{iii}}$ & $1.843(2)$ \\
\hline $\mathrm{Ca} 2-\mathrm{F}^{\mathrm{viii}}$ & $2.369(2)$ & $\mathrm{Al}-\mathrm{F} 3^{\mathrm{iii}}$ & $1.846(2)$ \\
\hline $\mathrm{F} 7-\mathrm{Ba}-\mathrm{F} 4^{\mathrm{i}}$ & $64.22(7)$ & $\mathrm{F}{ }^{\mathrm{iii}}-\mathrm{Ca} 2-\mathrm{F}^{\mathrm{viii}}$ & $100.95(11)$ \\
\hline $\mathrm{F} 7-\mathrm{Ba}-\mathrm{F} 1^{\mathrm{i}}$ & $158.04(7)$ & $\mathrm{F} 7^{\mathrm{vii}}-\mathrm{Ca} 2-\mathrm{F} 4$ & $139.26(9)$ \\
\hline $\mathrm{F} 4-\mathrm{Ba}-\mathrm{F} 1^{\mathrm{i}}$ & $97.60(7)$ & $\mathrm{F} 7^{\mathrm{i}}-\mathrm{Ca} 2-\mathrm{F} 4$ & $73.58(8)$ \\
\hline $\mathrm{F} 7-\mathrm{Ba}-\mathrm{F} 2^{\mathrm{ii}}$ & $89.56(7)$ & 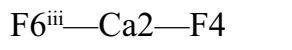 & $133.25(7)$ \\
\hline $\mathrm{F} 4^{\mathrm{i}}-\mathrm{Ba}-\mathrm{F} 2^{\mathrm{ii}}$ & $65.13(7)$ & 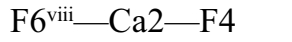 & $109.76(8)$ \\
\hline $\mathrm{F} 1^{\mathrm{i}}-\mathrm{Ba}-\mathrm{F} 2^{\mathrm{ii}}$ & $70.80(7)$ & $\mathrm{F} 7^{\mathrm{vii}}-\mathrm{Ca} 2-\mathrm{F} 4^{\mathrm{ix}}$ & $73.58(8)$ \\
\hline $\mathrm{F} 7-\mathrm{Ba}-\mathrm{F} 5^{\mathrm{iii}}$ & $102.89(6)$ & $\mathrm{F} 7^{\mathrm{i}}-\mathrm{Ca} 2-\mathrm{F} 4^{\mathrm{ix}}$ & $139.26(9)$ \\
\hline $\mathrm{F} 4^{\mathrm{i}}-\mathrm{Ba}-\mathrm{F} 5^{\mathrm{iii}}$ & $63.11(6)$ & $\mathrm{F} 6^{\mathrm{iii}}-\mathrm{Ca} 2-\mathrm{F} 4^{\mathrm{ix}}$ & $109.76(8)$ \\
\hline 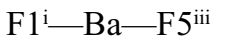 & $77.18(7)$ & $\mathrm{F} 6^{\mathrm{viii}}-\mathrm{Ca} 2-\mathrm{F} 4^{\mathrm{ix}}$ & $133.25(7)$ \\
\hline $\mathrm{F} 2^{\mathrm{ii}}-\mathrm{Ba}-\mathrm{F} 5^{\mathrm{iii}}$ & $113.14(6)$ & $\mathrm{F} 4-\mathrm{Ca} 2-\mathrm{F} 4^{\mathrm{ix}}$ & $73.00(12)$ \\
\hline $\mathrm{F} 7-\mathrm{Ba}-\mathrm{F} 5^{\mathrm{iv}}$ & $94.51(6)$ & $\mathrm{F}^{\mathrm{vii}}-\mathrm{Ca} 2-\mathrm{F}^{\mathrm{x}}$ & $86.30(7)$ \\
\hline 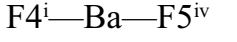 & $134.23(6)$ & $\mathrm{F} 7^{\mathrm{i}}-\mathrm{Ca} 2-\mathrm{F} 5^{\mathrm{x}}$ & $110.96(8)$ \\
\hline $\mathrm{F} 1^{\mathrm{i}}-\mathrm{Ba}-\mathrm{F} 5^{\mathrm{iv}}$ & $107.32(7)$ & $\mathrm{F} 6^{\mathrm{iii}}-\mathrm{Ca} 2-\mathrm{F}^{\mathrm{x}}$ & $165.39(7)$ \\
\hline $\mathrm{F} 2^{\mathrm{ii}}-\mathrm{Ba}-\mathrm{F} 5^{\mathrm{iv}}$ & $159.58(6)$ & $\mathrm{F} 6^{\mathrm{viii}}-\mathrm{Ca} 2-\mathrm{F}^{\mathrm{x}}$ & $70.35(7)$ \\
\hline $\mathrm{F} 5^{\mathrm{iii}}-\mathrm{Ba}-\mathrm{F} 5^{\mathrm{iv}}$ & $85.46(4)$ & $\mathrm{F} 4-\mathrm{Ca} 2-\mathrm{F}^{\mathrm{x}}$ & $61.35(7)$ \\
\hline $\mathrm{F} 7-\mathrm{Ba}-\mathrm{F}^{\mathrm{iv}}$ & $108.42(7)$ & $\mathrm{F} 4^{\mathrm{ix}}-\mathrm{Ca} 2-\mathrm{F} 5^{\mathrm{x}}$ & $71.46(7)$ \\
\hline $\mathrm{F} 4^{\mathrm{i}}-\mathrm{Ba}-\mathrm{F} 3^{\mathrm{iv}}$ & $168.26(6)$ & $\mathrm{F} 7^{\mathrm{vii}}-\mathrm{Ca} 2-\mathrm{F}^{\mathrm{iv}}$ & $110.96(8)$ \\
\hline $\mathrm{F} 1^{\mathrm{i}}-\mathrm{Ba}-\mathrm{F}^{\mathrm{iv}}$ & $87.26(7)$ & $\mathrm{F} 7^{\mathrm{i}}-\mathrm{Ca} 2-\mathrm{F}^{2 \mathrm{iv}}$ & $86.30(7)$ \\
\hline $\mathrm{F} 2^{\mathrm{ii}}-\mathrm{Ba}-\mathrm{F} 3^{\mathrm{iv}}$ & $106.93(6)$ & $\mathrm{F} 6^{\mathrm{iii}}-\mathrm{Ca} 2-\mathrm{F} 5^{\mathrm{iv}}$ & $70.35(7)$ \\
\hline 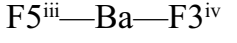 & $128.56(6)$ & 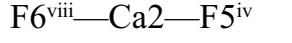 & $165.39(7)$ \\
\hline $\mathrm{F} 5^{\mathrm{iv}}-\mathrm{Ba}-\mathrm{F} 3^{\mathrm{iv}}$ & $52.84(6)$ & $\mathrm{F} 4-\mathrm{Ca} 2-\mathrm{F} 5^{\mathrm{iv}}$ & $71.46(7)$ \\
\hline $\mathrm{F} 7-\mathrm{Ba}-\mathrm{F} 1^{\mathrm{v}}$ & $58.57(7)$ & $\mathrm{F} 4^{\mathrm{ix}}-\mathrm{Ca} 2-\mathrm{F}^{\mathrm{iv}}$ & $61.35(7)$ \\
\hline $\mathrm{F} 4^{\mathrm{i}}-\mathrm{Ba}-\mathrm{F} 1^{\mathrm{v}}$ & $122.79(7)$ & $\mathrm{F} 5^{\mathrm{x}}-\mathrm{Ca} 2-\mathrm{F} 5^{\mathrm{iv}}$ & $120.53(9)$ \\
\hline 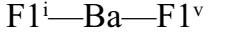 & $138.03(11)$ & $\mathrm{Fe} 1^{\mathrm{ix}}-\mathrm{Ca} 2-\mathrm{Ca} 1^{\mathrm{ix}}$ & 0.0 \\
\hline 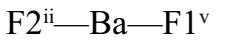 & $113.81(7)$ & $\mathrm{F} 4^{\mathrm{xii}}-\mathrm{Al}-\mathrm{F} 1$ & $175.02(12)$ \\
\hline $\mathrm{F} 5^{\mathrm{iii}}-\mathrm{Ba}-\mathrm{F} 1^{\mathrm{v}}$ & $128.75(7)$ & $\mathrm{F} 4^{\mathrm{xii}}-\mathrm{Al}-\mathrm{F}^{\mathrm{iv}}$ & 93.99 (12) \\
\hline $\mathrm{F} 5^{\mathrm{iv}}-\mathrm{Ba}-\mathrm{F} 1^{\mathrm{v}}$ & $53.35(7)$ & $\mathrm{F} 1-\mathrm{Al}-\mathrm{F} 6^{\mathrm{iv}}$ & $90.60(11)$ \\
\hline $\mathrm{F} 3^{\mathrm{iv}}-\mathrm{Ba}-\mathrm{F} 1^{\mathrm{v}}$ & $50.92(7)$ & $\mathrm{F} 4^{\mathrm{xii}}-\mathrm{Al}-\mathrm{F} 2^{\mathrm{iv}}$ & $93.23(11)$ \\
\hline $\mathrm{F} 7-\mathrm{Ba}-\mathrm{F} 3$ & $59.03(6)$ & $\mathrm{F} 1-\mathrm{Al}-\mathrm{F} 2^{\mathrm{iv}}$ & $88.30(12)$ \\
\hline $\mathrm{F} 4-\mathrm{Ba}-\mathrm{F} 3$ & $90.21(6)$ & $\mathrm{F} 6^{\mathrm{iv}}-\mathrm{Al}-\mathrm{F} 2^{\mathrm{iv}}$ & $94.65(11)$ \\
\hline $\mathrm{F} 1 \mathrm{i}-\mathrm{Ba}-\mathrm{F} 3$ & $111.82(7)$ & $\mathrm{F} 4^{\mathrm{xii}}-\mathrm{Al}-\mathrm{F} 5^{\mathrm{iii}}$ & $87.88(10)$ \\
\hline $\mathrm{F} 2^{\mathrm{ii}}-\mathrm{Ba}-\mathrm{F} 3$ & $52.19(6)$ & $\mathrm{F} 1-\mathrm{Al}-\mathrm{F} 5^{\mathrm{iii}}$ & $90.20(12)$ \\
\hline 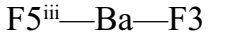 & $153.12(6)$ & $\mathrm{F} 6^{\mathrm{iv}}-\mathrm{Al}-\mathrm{F} 5^{\mathrm{iii}}$ & $90.11(11)$ \\
\hline
\end{tabular}




\begin{tabular}{|c|c|c|c|}
\hline $\mathrm{F} 5^{\mathrm{iv}}-\mathrm{Ba}-\mathrm{F} 3$ & $113.88(6)$ & $\mathrm{F} 2^{\mathrm{iv}}-\mathrm{Al}-\mathrm{F} 5^{\mathrm{iii}}$ & $175.02(11)$ \\
\hline $\mathrm{F} 3^{\mathrm{iv}}-\mathrm{Ba}-\mathrm{F} 3$ & $78.06(7)$ & $\mathrm{F} 4^{\mathrm{xii}}-\mathrm{Al}-\mathrm{F} 3^{\mathrm{iii}}$ & $88.87(11)$ \\
\hline $\mathrm{F} 1 \mathrm{v}-\mathrm{Ba}-\mathrm{F} 3$ & $61.81(7)$ & $\mathrm{F} 1-\mathrm{Al}-\mathrm{F} 3^{\mathrm{iii}}$ & $86.45(11)$ \\
\hline $\mathrm{F} 7-\mathrm{Ba}-\mathrm{F} 6^{\mathrm{iii}}$ & $149.96(6)$ & $\mathrm{F} 6^{\mathrm{iv}}-\mathrm{Al}-\mathrm{F} 3^{\mathrm{iii}}$ & $176.00(12)$ \\
\hline $\mathrm{F} 4 \mathrm{i}-\mathrm{Ba}-\mathrm{F} 6^{\mathrm{iii}}$ & $127.50(6)$ & $\mathrm{F} 2^{\mathrm{iv}}-\mathrm{Al}-\mathrm{F} 3^{\mathrm{iii}}$ & $87.98(11)$ \\
\hline $\mathrm{F} 1^{\mathrm{i}}-\mathrm{Ba}-\mathrm{F} 6^{\mathrm{iii}}$ & $50.94(6)$ & $\mathrm{F} 5^{\mathrm{iii}}-\mathrm{Al}-\mathrm{F}^{\mathrm{iii}}$ & $87.19(10)$ \\
\hline $\mathrm{F} 2^{\mathrm{ii}}-\mathrm{Ba}-\mathrm{F}^{\mathrm{iii}}$ & $120.41(6)$ & $\mathrm{Al}-\mathrm{F} 1-\mathrm{Ba}^{\mathrm{v}}$ & $114.10(11)$ \\
\hline 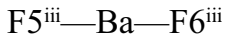 & $68.78(5)$ & $\mathrm{Al}-\mathrm{F} 1-\mathrm{Ba}^{\mathrm{i}}$ & $96.30(9)$ \\
\hline $\mathrm{F} 5^{\mathrm{iv}}-\mathrm{Ba}-\mathrm{F} 6^{\mathrm{iii}}$ & $56.89(6)$ & $\mathrm{Ba}^{\mathrm{v}}-\mathrm{F} 1-\mathrm{Ba}^{\mathrm{i}}$ & $138.03(11)$ \\
\hline $\mathrm{F} 3^{\mathrm{iv}}-\mathrm{Ba}-\mathrm{F} 6^{\mathrm{iii}}$ & $63.59(6)$ & $\mathrm{Al}-\mathrm{F} 1-\mathrm{Ba}^{\mathrm{iii}}$ & $90.11(11)$ \\
\hline $\mathrm{F} 1^{\mathrm{v}}-\mathrm{Ba}-\mathrm{F} 6^{\mathrm{iii}}$ & $103.02(6)$ & $\mathrm{Ba}^{\mathrm{v}}-\mathrm{F} 1-\mathrm{Ba}^{\mathrm{iii}}$ & $111.43(8)$ \\
\hline $\mathrm{F} 3-\mathrm{Ba}-\mathrm{F} 6^{\mathrm{iii}}$ & $137.04(6)$ & $\mathrm{Ba}^{\mathrm{i}}-\mathrm{F} 1-\mathrm{Ba}^{\mathrm{iii}}$ & $95.98(7)$ \\
\hline $\mathrm{F} 7-\mathrm{Ba}-\mathrm{F} 6^{\mathrm{iv}}$ & $58.76(6)$ & $\mathrm{Al}^{\mathrm{iv}}-\mathrm{F} 2-\mathrm{Ca} 1^{\mathrm{xiii}}$ & $136.08(11)$ \\
\hline $\mathrm{F} 4-\mathrm{Ba}-\mathrm{F}^{\mathrm{i}}{ }^{\mathrm{iv}}$ & $67.12(6)$ & $\mathrm{Al}^{\mathrm{iv}}-\mathrm{F} 2-\mathrm{Fe}^{\mathrm{xiii}}$ & $136.08(11)$ \\
\hline 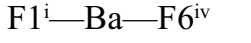 & $127.71(7)$ & $\mathrm{Ca} 1^{\mathrm{xiii}}-\mathrm{F} 2-\mathrm{Fe} 1^{\mathrm{xiii}}$ & 0.0 \\
\hline 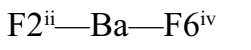 & $130.73(6)$ & $\mathrm{Al}^{\mathrm{iv}}-\mathrm{F} 2-\mathrm{Ba}^{\mathrm{xiv}}$ & $106.09(9)$ \\
\hline $\mathrm{F} 5^{\mathrm{iii}-}-\mathrm{Ba}-\mathrm{F} 6^{\mathrm{iv}}$ & $50.92(6)$ & $\mathrm{Ca} 1^{\mathrm{xiii}}-\mathrm{F} 2-\mathrm{Ba}^{\mathrm{xiv}}$ & $117.51(8)$ \\
\hline $\mathrm{F} 5^{\text {iv }}-\mathrm{Ba}-\mathrm{F} 6^{\mathrm{iv}}$ & $67.24(5)$ & $\mathrm{Fe} 1^{\mathrm{xii}}-\mathrm{F} 2-\mathrm{Ba}^{\mathrm{xiv}}$ & $117.51(8)$ \\
\hline $\mathrm{F} 3^{\mathrm{iv}}-\mathrm{Ba}-\mathrm{F} 6^{\mathrm{iv}}$ & $118.00(6)$ & $\mathrm{Al}^{\mathrm{vi}}-\mathrm{F} 3-\mathrm{Ca} 1^{\mathrm{v}}$ & $125.67(12)$ \\
\hline $\mathrm{F} 1^{\mathrm{v}}-\mathrm{Ba}-\mathrm{F} 6^{\mathrm{iv}}$ & $82.68(7)$ & $\mathrm{Al}^{\mathrm{vi}}-\mathrm{F} 3-\mathrm{Fe} 1^{\mathrm{v}}$ & $125.67(12)$ \\
\hline $\mathrm{F} 3-\mathrm{Ba}-\mathrm{F} 6^{\mathrm{iv}}$ & $117.62(5)$ & $\mathrm{Ca} 1^{\mathrm{v}}-\mathrm{F} 3-\mathrm{Fe}^{\mathrm{v}}$ & 0.0 \\
\hline $\mathrm{F} 6^{\mathrm{iii}-}-\mathrm{Ba}-\mathrm{F}^{\mathrm{iv}}$ & $97.89(5)$ & $\mathrm{Al}^{\mathrm{vi}}-\mathrm{F} 3-\mathrm{Ba}^{\mathrm{iv}}$ & $94.81(9)$ \\
\hline $\mathrm{F} 7-\mathrm{Ba}-\mathrm{F} 1^{\mathrm{vi}}$ & $105.96(6)$ & $\mathrm{Ca} 1^{\mathrm{v}}-\mathrm{F} 3-\mathrm{Ba}^{\mathrm{iv}}$ & $131.32(9)$ \\
\hline $\mathrm{F} 4-\mathrm{Ba}-\mathrm{F} 1^{\mathrm{vi}}$ & $113.22(7)$ & $\mathrm{Fe}^{\mathrm{v}}-\mathrm{F} 3-\mathrm{Ba}^{\mathrm{iv}}$ & $131.32(9)$ \\
\hline $\mathrm{F} 1^{\mathrm{i}}-\mathrm{Ba}-\mathrm{F} 1^{\mathrm{vi}}$ & $68.57(8)$ & $\mathrm{Al}^{\mathrm{vi}}-\mathrm{F} 3-\mathrm{Ba}$ & $97.28(9)$ \\
\hline 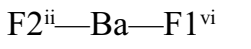 & $48.35(6)$ & $\mathrm{Ca}^{\mathrm{v}}-\mathrm{F} 3-\mathrm{Ba}$ & $98.63(7)$ \\
\hline $\mathrm{F} 5^{\mathrm{iii}}-\mathrm{Ba}-\mathrm{F} 1^{\mathrm{vi}}$ & $144.90(6)$ & $\mathrm{Fe} 1^{v}-\mathrm{F} 3-\mathrm{Ba}$ & $98.63(7)$ \\
\hline $\mathrm{F} 5^{\mathrm{iv}}-\mathrm{Ba}-\mathrm{F} 1^{\mathrm{vi}}$ & $111.48(6)$ & $\mathrm{Ba}^{\mathrm{iv}}-\mathrm{F} 3-\mathrm{Ba}$ & $101.94(7)$ \\
\hline $\mathrm{F} 3^{\mathrm{iv}}-\mathrm{Ba}-\mathrm{F} 1^{\mathrm{vi}}$ & $58.64(6)$ & $\mathrm{Al}^{\mathrm{xv}}-\mathrm{F} 4-\mathrm{Ca} 2$ & $107.90(10)$ \\
\hline $\mathrm{F} 1^{\mathrm{v}}-\mathrm{Ba}-\mathrm{F} 1^{\mathrm{vi}}$ & $84.02(7)$ & $\mathrm{Al}^{\mathrm{xv}}-\mathrm{F} 4-\mathrm{Ba}^{\mathrm{v}}$ & $142.62(11)$ \\
\hline $\mathrm{F} 3-\mathrm{Ba}-\mathrm{F} 1^{\mathrm{vi}}$ & $46.95(6)$ & $\mathrm{Ca} 2-\mathrm{F} 4-\mathrm{Ba}^{\mathrm{v}}$ & $109.17(8)$ \\
\hline 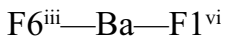 & $94.27(6)$ & $\mathrm{Al}^{\mathrm{vi}}-\mathrm{F} 5-\mathrm{Ca} 2^{\mathrm{iv}}$ & $99.49(9)$ \\
\hline $\mathrm{F} 6^{\mathrm{iv}}-\mathrm{Ba}-\mathrm{F} 1^{\mathrm{vi}}$ & $163.69(5)$ & $\mathrm{Al}^{\mathrm{vi}}-\mathrm{F} 5-\mathrm{Ba}^{\mathrm{vi}}$ & $116.54(9)$ \\
\hline $\mathrm{F} 7^{\mathrm{x}}-\mathrm{Fe} 1-\mathrm{F} 2^{\mathrm{xi}}$ & $85.92(8)$ & $\mathrm{Ca} 2^{\mathrm{iv}}-\mathrm{F} 5-\mathrm{Ba}^{\mathrm{vi}}$ & $103.29(7)$ \\
\hline $\mathrm{F} 7^{\mathrm{i}}-\mathrm{Fe} 1-\mathrm{F} 2^{\mathrm{xi}}$ & $94.08(8)$ & $\mathrm{Al}^{\mathrm{vi}}-\mathrm{F} 5-\mathrm{Ba}^{\mathrm{iv}}$ & $96.93(8)$ \\
\hline $\mathrm{F} 7^{\mathrm{x}}-\mathrm{Fe} 1-\mathrm{F} 2^{\mathrm{viii}}$ & $94.08(8)$ & $\mathrm{Ca} 2^{\mathrm{iv}}-\mathrm{F} 5-\mathrm{Ba}^{\mathrm{iv}}$ & $117.45(8)$ \\
\hline $\mathrm{F} 7^{\mathrm{i}}-\mathrm{Fe} 1-\mathrm{F}^{\mathrm{viii}}$ & $85.92(8)$ & $\mathrm{Ba}^{\mathrm{vi}}-\mathrm{F} 5-\mathrm{Ba}^{\mathrm{iv}}$ & $121.50(7)$ \\
\hline $\mathrm{F}^{\mathrm{x}}-\mathrm{Fe} 1-\mathrm{F}^{\mathrm{x}}$ & $82.86(8)$ & $\mathrm{Al}^{\mathrm{iv}}-\mathrm{F} 6-\mathrm{Ca}^{\text {viii }}$ & $133.22(12)$ \\
\hline $\mathrm{F} 7^{\mathrm{i}}-\mathrm{Fe} 1-\mathrm{F}^{\mathrm{x}}$ & $97.14(8)$ & $\mathrm{Al}^{\mathrm{iv}}-\mathrm{F} 6-\mathrm{Ba}^{\mathrm{vi}}$ & $100.10(9)$ \\
\hline $\mathrm{F} 2^{\mathrm{xi}}-\mathrm{Fe} 1-\mathrm{F}^{\mathrm{x}}$ & $95.71(8)$ & 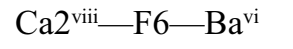 & $113.52(8)$ \\
\hline $\mathrm{F} 2^{\mathrm{viii}-\mathrm{Fe} 1-\mathrm{F}^{\mathrm{x}}}$ & $84.29(8)$ & $\mathrm{Al}^{\mathrm{iv}}-\mathrm{F} 6-\mathrm{Ba}^{\mathrm{iv}}$ & $102.40(9)$ \\
\hline $\mathrm{F}^{\mathrm{x}}-\mathrm{Fe} 1-\mathrm{F}^{\mathrm{i}}$ & $97.14(8)$ & $\mathrm{Ca}^{\text {viii }}-\mathrm{F} 6-\mathrm{Ba}^{\mathrm{iv}}$ & $101.02(7)$ \\
\hline $\mathrm{F} 7^{\mathrm{i}}-\mathrm{Fe} 1-\mathrm{F} 3^{\mathrm{i}}$ & $82.86(8)$ & $\mathrm{Ba}^{\mathrm{vi}}-\mathrm{F} 6-\mathrm{Ba}^{\mathrm{iv}}$ & $102.46(6)$ \\
\hline $\mathrm{F} 2^{\mathrm{xi}}-\mathrm{Fe} 1-\mathrm{F}^{\mathrm{i}}$ & $84.29(8)$ & $\mathrm{Ca}^{\mathrm{v}}-\mathrm{F} 7-\mathrm{Fe}^{\mathrm{v}}$ & 0.0 \\
\hline $\mathrm{F} 2^{\mathrm{viii}}-\mathrm{Fe} 1-\mathrm{F} 3^{\mathrm{i}}$ & $95.71(8)$ & $\mathrm{Ca} 1^{\mathrm{v}}-\mathrm{F} 7-\mathrm{Ca} 2^{\mathrm{xvi}}$ & $120.98(11)$ \\
\hline $\mathrm{F} 7^{\mathrm{vii}}-\mathrm{Ca} 2-\mathrm{F}^{\mathrm{i}}$ & $145.63(12)$ & $\mathrm{Fe} 1^{\mathrm{v}}-\mathrm{F} 7-\mathrm{Ca} 2^{\mathrm{xvi}}$ & $120.98(11)$ \\
\hline $\mathrm{F}^{\mathrm{vii}}-\mathrm{Ca} 2-\mathrm{F}^{\mathrm{iii}}$ & $80.29(8)$ & $\mathrm{Ca} 1^{\mathrm{v}}-\mathrm{F} 7-\mathrm{Ba}$ & $113.91(9)$ \\
\hline $\mathrm{F}^{\mathrm{i}}-\mathrm{Ca} 2-\mathrm{F} 6^{\mathrm{iii}}$ & $78.02(8)$ & $\mathrm{Fe} 1^{\mathrm{v}}-\mathrm{F} 7-\mathrm{Ba}$ & $113.91(9)$ \\
\hline
\end{tabular}




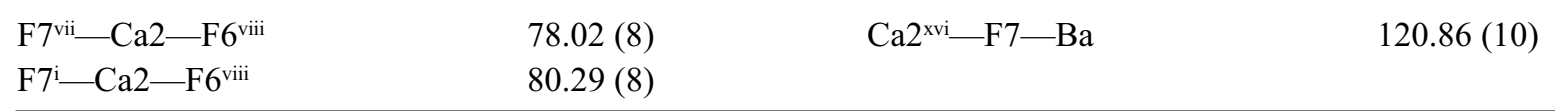

Symmetry codes: (i) $-x+1 / 2, y+1 / 2,-z+1 / 2$; (ii) $-x+1 / 2, y+1 / 2,-z+3 / 2$; (iii) $x,-y+1, z-1 / 2$; (iv) $-x+1 / 2,-y+1 / 2,-z+1$; (v) $-x+1 / 2, y-1 / 2,-z+1 / 2$; (vi) $x$, $-y+1, z+1 / 2$; (vii) $x-1 / 2, y+1 / 2, z$; (viii) $-x,-y+1,-z+1$; (ix) $-x, y,-z+1 / 2$; (x) $x-1 / 2,-y+1 / 2, z-1 / 2$; (xi) $x, y, z-1$; (xii) $x+1 / 2, y+1 / 2, z$; (xiii) $x, y, z+1$; (xiv) $-x+1 / 2, y-1 / 2,-z+3 / 2$; (xv) $x-1 / 2, y-1 / 2, z$; (xvi) $x+1 / 2, y-1 / 2, z$.

\section{(modelII)}

\section{Crystal data}

$$
\begin{aligned}
& \mathrm{Al}_{2} \mathrm{Ba}_{2} \mathrm{CaF}_{14} \mathrm{Fe}_{0.90} \\
& M_{r}=684.98 \\
& \text { Monoclinic, } C 2 / c \\
& \text { Hall symbol: }-\mathrm{C} 2 \mathrm{yc} \\
& a=13.7387(12) \AA \\
& b=5.2701(5) \AA \\
& c=14.759(3) \AA \\
& \beta=92.074(14)^{\circ} \\
& V=1067.9(2) \AA^{3} \\
& Z=4
\end{aligned}
$$

\section{Data collection}

Nonius CAD-4 four-circle diffractometer

Radiation source: fine-focus sealed tube Graphite monochromator $\omega / 2 \theta$ scans

Absorption correction: $\psi$ scan

(North et al., 1968)

$T_{\min }=0.329, T_{\max }=0.901$

5922 measured reflections

\section{Refinement}

Refinement on $F^{2}$

Least-squares matrix: full

$R\left[F^{2}>2 \sigma\left(F^{2}\right)\right]=0.032$

$w R\left(F^{2}\right)=0.078$

$S=1.10$

1564 reflections

96 parameters

0 restraints
$F(000)=1240$

$D_{\mathrm{x}}=4.260 \mathrm{Mg} \mathrm{m}^{-3}$

Mo $K \alpha$ radiation, $\lambda=0.71073 \AA$

Cell parameters from 25 reflections

$\theta=16.0-28.7^{\circ}$

$\mu=9.33 \mathrm{~mm}^{-1}$

$T=293 \mathrm{~K}$

Lath, colourless

$0.43 \times 0.11 \times 0.07 \mathrm{~mm}$

1564 independent reflections

1490 reflections with $I>2 \sigma(I)$

$R_{\text {int }}=0.055$

$\theta_{\text {max }}=30.0^{\circ}, \theta_{\min }=2.8^{\circ}$

$h=-19 \rightarrow 19$

$k=-7 \rightarrow 7$

$l=-20 \rightarrow 20$

3 standard reflections every $240 \mathrm{~min}$ intensity decay: none

Primary atom site location: isomorphous structure methods

$w=1 /\left[\sigma^{2}\left(F_{\mathrm{o}}^{2}\right)+(0.0532 P)^{2}+1.6437 P\right]$ where $P=\left(F_{\mathrm{o}}{ }^{2}+2 F_{\mathrm{c}}{ }^{2}\right) / 3$

$(\Delta / \sigma)_{\max }=0.001$

$\Delta \rho_{\max }=2.32 \mathrm{e} \AA^{-3}$

$\Delta \rho_{\min }=-2.03$ e $\AA^{-3}$

Extinction correction: SHELXL, $\mathrm{Fc}^{*}=\mathrm{kFc}\left[1+0.001 \times \mathrm{Fc}^{2} \lambda^{3} / \sin (2 \theta)\right]^{-1 / 4}$

Extinction coefficient: 0.0022 (2)

\section{Special details}

Geometry. All e.s.d.'s (except the e.s.d. in the dihedral angle between two 1.s. planes) are estimated using the full covariance matrix. The cell e.s.d.'s are taken into account individually in the estimation of e.s.d.'s in distances, angles and torsion angles; correlations between e.s.d.'s in cell parameters are only used when they are defined by crystal symmetry. An approximate (isotropic) treatment of cell e.s.d.'s is used for estimating e.s.d.'s involving 1.s. planes.

Refinement. Refinement of $F^{2}$ against ALL reflections. The weighted $R$-factor $w R$ and goodness of fit $S$ are based on $F^{2}$, conventional $R$-factors $R$ are based on $F$, with $F$ set to zero for negative $F^{2}$. The threshold expression of $F^{2}>\sigma\left(F^{2}\right)$ is used only for calculating $R$-factors(gt) etc. and is not relevant to the choice of reflections for refinement. $R$-factors based on $F^{2}$ are statistically about twice as large as those based on $F$, and $R$ - factors based on ALL data will be even larger. 


\section{supporting information}

Fractional atomic coordinates and isotropic or equivalent isotropic displacement parameters $\left(\AA^{2}\right)$

\begin{tabular}{llllll}
\hline & $x$ & $y$ & $z$ & $U_{\text {iso }} * / U_{\text {eq }}$ & Occ. $(<1)$ \\
\hline $\mathrm{Ba}$ & $0.308719(14)$ & $0.46574(4)$ & $0.379962(12)$ & $0.01408(12)$ & \\
$\mathrm{Ca}$ & 0.0000 & $0.44617(15)$ & 0.2500 & $0.01239(19)$ & \\
$\mathrm{Al}$ & $0.38008(8)$ & $0.50390(18)$ & $0.12340(7)$ & $0.0111(2)$ & \\
$\mathrm{Fe}$ & 0.0000 & 0.5000 & 0.0000 & $0.0114(3)$ & $0.901(5)$ \\
$\mathrm{F} 1$ & $0.25596(18)$ & $0.4521(4)$ & $0.0896(2)$ & $0.0240(5)$ & \\
$\mathrm{F} 2$ & $0.08572(15)$ & $0.1721(4)$ & $0.97492(13)$ & $0.0184(4)$ & \\
$\mathrm{F} 3$ & $0.37360(16)$ & $0.2100(4)$ & $0.55132(14)$ & $0.0197(4)$ & \\
$\mathrm{F} 4$ & $0.00267(15)$ & $0.0837(4)$ & $0.15434(15)$ & $0.0190(4)$ & \\
$\mathrm{F} 5$ & $0.34178(14)$ & $0.2932(4)$ & $0.71742(13)$ & $0.0157(4)$ & \\
$\mathrm{F} 6$ & $0.12185(16)$ & $0.2677(4)$ & $0.80392(14)$ & $0.0207(4)$ & \\
F7 & $0.43372(16)$ & $0.0715(4)$ & $0.37867(16)$ & $0.0208(4)$ & \\
& & & & &
\end{tabular}

Atomic displacement parameters $\left(\AA^{2}\right)$

\begin{tabular}{lllllll}
\hline & $U^{11}$ & $U^{22}$ & $U^{33}$ & $U^{12}$ & $U^{13}$ & $U^{23}$ \\
\hline $\mathrm{Ba}$ & $0.01226(16)$ & $0.01399(16)$ & $0.01592(15)$ & $-0.00069(5)$ & $-0.00023(9)$ & $0.00074(5)$ \\
$\mathrm{Ca}$ & $0.0131(4)$ & $0.0124(4)$ & $0.0118(4)$ & 0.000 & $0.0019(3)$ & 0.000 \\
$\mathrm{Al}$ & $0.0114(5)$ & $0.0096(3)$ & $0.0123(5)$ & $-0.0007(3)$ & $-0.0003(4)$ & $0.0005(3)$ \\
$\mathrm{Fe}$ & $0.0098(4)$ & $0.0115(3)$ & $0.0128(4)$ & $0.0004(2)$ & $-0.0005(3)$ & $-0.0030(2)$ \\
$\mathrm{F} 1$ & $0.0129(11)$ & $0.0219(11)$ & $0.0366(14)$ & $-0.0017(8)$ & $-0.0068(10)$ & $-0.0007(9)$ \\
$\mathrm{F} 2$ & $0.0219(10)$ & $0.0184(9)$ & $0.0148(8)$ & $0.0032(8)$ & $-0.0016(7)$ & $-0.0026(7)$ \\
F3 & $0.0270(11)$ & $0.0140(8)$ & $0.0184(9)$ & $-0.0004(8)$ & $0.0027(8)$ & $-0.0031(7)$ \\
F4 & $0.0100(9)$ & $0.0247(9)$ & $0.0224(10)$ & $-0.0013(8)$ & $0.0000(7)$ & $-0.0086(8)$ \\
F5 & $0.0156(9)$ & $0.0172(8)$ & $0.0145(8)$ & $-0.0038(7)$ & $0.0027(6)$ & $0.0004(7)$ \\
F6 & $0.0221(10)$ & $0.0160(8)$ & $0.0243(10)$ & $0.0037(8)$ & $0.0036(8)$ & $0.0076(8)$ \\
F7 & $0.0182(11)$ & $0.0238(9)$ & $0.0207(10)$ & $0.0008(8)$ & $0.0053(8)$ & $-0.0067(8)$ \\
& & & & & &
\end{tabular}

Geometric parameters $\left(\AA,{ }^{\circ}\right)$

\begin{tabular}{|c|c|c|c|}
\hline $\mathrm{Ba}-\mathrm{F} 7$ & $2.696(2)$ & $\mathrm{Ca}-\mathrm{F} 4$ & $2.376(2)$ \\
\hline $\mathrm{Ba}-\mathrm{F} 4^{\mathrm{i}}$ & $2.730(2)$ & $\mathrm{Ca}-\mathrm{F} 4^{\mathrm{ix}}$ & $2.376(2)$ \\
\hline $\mathrm{Ba}-\mathrm{F} 1^{\mathrm{i}}$ & $2.755(2)$ & $\mathrm{Ca}-\mathrm{F}^{\mathrm{x}}$ & $2.544(2)$ \\
\hline $\mathrm{Ba}-\mathrm{F}^{\mathrm{ii}}$ & $2.766(2)$ & $\mathrm{Ca}-\mathrm{F}^{\mathrm{iv}}$ & $2.544(2)$ \\
\hline $\mathrm{Ba}-\mathrm{F}^{\mathrm{iii}}$ & $2.767(2)$ & $\mathrm{Fe}-\mathrm{F} 7^{\mathrm{i}}$ & $2.015(2)$ \\
\hline $\mathrm{Ba}-\mathrm{F}^{\text {iv }}$ & $2.826(2)$ & $\mathrm{Fe}-\mathrm{F}^{\mathrm{x}}$ & $2.015(2)$ \\
\hline $\mathrm{Ba}-\mathrm{F}^{\mathrm{iv}}$ & $2.889(2)$ & $\mathrm{Fe}-\mathrm{F} 2^{\text {viii }}$ & $2.131(2)$ \\
\hline $\mathrm{Ba}-\mathrm{F} 1^{\mathrm{v}}$ & $2.889(2)$ & $\mathrm{Fe}-\mathrm{F} 2^{\mathrm{xi}}$ & $2.131(2)$ \\
\hline $\mathrm{Ba}-\mathrm{F} 3$ & $2.974(2)$ & $\mathrm{Fe}-\mathrm{F}^{\mathrm{x}}$ & $2.216(2)$ \\
\hline $\mathrm{Ba}-\mathrm{F} 6^{\mathrm{iii}}$ & $3.101(2)$ & $\mathrm{Fe}-\mathrm{F} 3^{\mathrm{i}}$ & $2.216(2)$ \\
\hline $\mathrm{Ba}-\mathrm{F} 6^{\mathrm{iv}}$ & 3.159 (2) & $\mathrm{Al}-\mathrm{F} 4^{\mathrm{xii}}$ & $1.779(2)$ \\
\hline $\mathrm{Ba}-\mathrm{F} 1^{\mathrm{vi}}$ & $3.232(3)$ & $\mathrm{Al}-\mathrm{F} 1$ & $1.780(3)$ \\
\hline $\mathrm{Ca}-\mathrm{F}^{\mathrm{vii}}$ & $2.235(2)$ & $\mathrm{Al}-\mathrm{F} 6^{\mathrm{iv}}$ & $1.790(2)$ \\
\hline $\mathrm{Ca}-\mathrm{F} 7^{\mathrm{i}}$ & $2.235(2)$ & $\mathrm{Al}-\mathrm{F}^{2 \mathrm{iv}}$ & 1.799 (2) \\
\hline $\mathrm{Ca}-\mathrm{F} 6^{\mathrm{iii}}$ & $2.369(2)$ & $\mathrm{Al}-\mathrm{F}^{5 \mathrm{iii}}$ & $1.843(2)$ \\
\hline $\mathrm{Ca}-\mathrm{F} 6^{\text {viii }}$ & $2.369(2)$ & $\mathrm{Al}-\mathrm{F}^{3 \mathrm{iii}}$ & $1.846(2)$ \\
\hline
\end{tabular}




\begin{tabular}{|c|c|c|c|}
\hline $\mathrm{F} 7-\mathrm{Ba}-\mathrm{F} 4^{\mathrm{i}}$ & $64.22(7)$ & $\mathrm{F} 7^{\mathrm{i}}-\mathrm{Ca}-\mathrm{F} 4^{\mathrm{ix}}$ & $139.26(9)$ \\
\hline $\mathrm{F} 7-\mathrm{Ba}-\mathrm{F} 1^{\mathrm{i}}$ & $158.04(7)$ & $\mathrm{F} 6^{\mathrm{iii}}-\mathrm{Ca}-\mathrm{F} 4^{\mathrm{ix}}$ & $109.75(8)$ \\
\hline $\mathrm{F} 4^{\mathrm{i}}-\mathrm{Ba}-\mathrm{F} 1^{\mathrm{i}}$ & $97.60(7)$ & $\mathrm{F} 6^{\mathrm{viii}}-\mathrm{Ca}-\mathrm{F} 4^{\mathrm{ix}}$ & $133.25(7)$ \\
\hline $\mathrm{F} 7-\mathrm{Ba}-\mathrm{F} 2^{\mathrm{ii}}$ & $89.56(7)$ & $\mathrm{F} 4-\mathrm{Ca}-\mathrm{F} 4^{\mathrm{ix}}$ & $73.01(12)$ \\
\hline $\mathrm{F} 4-\mathrm{Ba}-\mathrm{F} 2^{\mathrm{ii}}$ & $65.12(7)$ & $\mathrm{F} 7^{\mathrm{vii}}-\mathrm{Ca}-\mathrm{F}^{\mathrm{x}}$ & $86.30(7)$ \\
\hline $\mathrm{F} 1^{\mathrm{i}}-\mathrm{Ba}-\mathrm{F} 2^{\mathrm{ii}}$ & $70.80(7)$ & $\mathrm{F} 7^{\mathrm{i}}-\mathrm{Ca}-\mathrm{F} 5^{\mathrm{x}}$ & $110.95(8)$ \\
\hline $\mathrm{F} 7-\mathrm{Ba}-\mathrm{F} 5^{\mathrm{iii}}$ & $102.89(6)$ & $\mathrm{F} 6^{\mathrm{iii}}-\mathrm{Ca}-\mathrm{F}^{\mathrm{x}}$ & $165.38(7)$ \\
\hline $\mathrm{F} 4^{\mathrm{i}}-\mathrm{Ba}-\mathrm{F} 5^{\text {iii }}$ & $63.12(6)$ & $\mathrm{F} 6^{\mathrm{viii}}-\mathrm{Ca}-\mathrm{F}^{\mathrm{x}}$ & $70.34(7)$ \\
\hline $\mathrm{F} 1^{\mathrm{i}}-\mathrm{Ba}-\mathrm{F} 5^{\mathrm{iii}}$ & $77.18(7)$ & $\mathrm{F} 4-\mathrm{Ca}-\mathrm{F} 5^{\mathrm{x}}$ & $61.35(7)$ \\
\hline 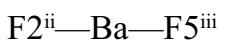 & $113.14(6)$ & $\mathrm{F} 4^{\mathrm{ix}}-\mathrm{Ca}-\mathrm{F} 5^{\mathrm{x}}$ & $71.47(7)$ \\
\hline $\mathrm{F} 7-\mathrm{Ba}-\mathrm{F} 5^{\text {iv }}$ & $94.51(6)$ & $\mathrm{F} 7^{\mathrm{vii}}-\mathrm{Ca}-\mathrm{F}^{\mathrm{iv}}$ & $110.95(7)$ \\
\hline $\mathrm{F} 4^{\mathrm{i}}-\mathrm{Ba}-\mathrm{F} 5^{\mathrm{iv}}$ & $134.23(6)$ & $\mathrm{F}^{\mathrm{i}}-\mathrm{Ca}-\mathrm{F} 5^{\mathrm{iv}}$ & $86.30(7)$ \\
\hline $\mathrm{F} 1^{\mathrm{i}}-\mathrm{Ba}-\mathrm{F} 5^{\mathrm{iv}}$ & $107.33(6)$ & $\mathrm{F} 6^{\mathrm{iii}}-\mathrm{Ca}-\mathrm{F}^{\mathrm{iv}}$ & $70.34(7)$ \\
\hline 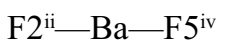 & $159.58(6)$ & $\mathrm{F} 6^{\mathrm{viii}}-\mathrm{Ca}-\mathrm{F}^{\mathrm{iv}}$ & $165.38(7)$ \\
\hline 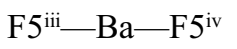 & $85.46(4)$ & $\mathrm{F} 4-\mathrm{Ca}-\mathrm{F} 5^{\text {iv }}$ & $71.47(7)$ \\
\hline $\mathrm{F} 7-\mathrm{Ba}-\mathrm{F} 3^{\mathrm{iv}}$ & $108.42(6)$ & $\mathrm{F} 4^{\mathrm{ix}}-\mathrm{Ca}-\mathrm{F} 5^{\mathrm{iv}}$ & $61.35(7)$ \\
\hline $\mathrm{F} 4^{\mathrm{i}}-\mathrm{Ba}-\mathrm{F}^{\mathrm{iv}}$ & $168.26(6)$ & $\mathrm{F} 5^{\mathrm{x}}-\mathrm{Ca}-\mathrm{F} 5^{\mathrm{iv}}$ & $120.54(9)$ \\
\hline $\mathrm{F} 1^{\mathrm{i}}-\mathrm{Ba}-\mathrm{F} 3^{\mathrm{iv}}$ & $87.26(7)$ & $\mathrm{F} 4^{\mathrm{xii}}-\mathrm{Al}-\mathrm{F} 1$ & $175.02(12)$ \\
\hline 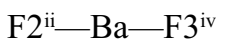 & $106.93(6)$ & $\mathrm{F} 4^{\mathrm{xii}}-\mathrm{Al}-\mathrm{F} 6^{\mathrm{iv}}$ & $94.00(11)$ \\
\hline 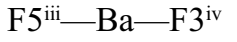 & $128.56(6)$ & $\mathrm{F} 1-\mathrm{Al}-\mathrm{F} 6^{\mathrm{iv}}$ & $90.60(11)$ \\
\hline $\mathrm{F} 5^{\mathrm{iv}}-\mathrm{Ba}-\mathrm{F} 3^{\mathrm{iv}}$ & $52.85(6)$ & $\mathrm{F} 4^{\mathrm{xii}}-\mathrm{Al}-\mathrm{F} 2^{\mathrm{iv}}$ & $93.23(11)$ \\
\hline $\mathrm{F} 7-\mathrm{Ba}-\mathrm{F} 1^{\mathrm{v}}$ & $58.57(7)$ & $\mathrm{F} 1-\mathrm{Al}-\mathrm{F} 2^{\mathrm{iv}}$ & $88.31(12)$ \\
\hline $\mathrm{F} 4^{\mathrm{i}}-\mathrm{Ba}-\mathrm{F} 1^{\mathrm{v}}$ & $122.79(7)$ & $\mathrm{F} 6^{\mathrm{iv}}-\mathrm{Al}-\mathrm{F} 2^{\mathrm{iv}}$ & $94.65(11)$ \\
\hline 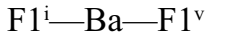 & $138.03(11)$ & $\mathrm{F} 4^{\mathrm{xii}}-\mathrm{Al}-\mathrm{F} 5^{\mathrm{iii}}$ & $87.88(10)$ \\
\hline $\mathrm{F} 2^{\mathrm{ii}-}-\mathrm{Ba}-\mathrm{F} 1^{\mathrm{v}}$ & $113.81(7)$ & $\mathrm{F} 1-\mathrm{Al}-\mathrm{F} 5^{\mathrm{iii}}$ & $90.18(12)$ \\
\hline 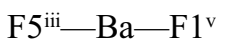 & $128.76(7)$ & $\mathrm{F} 6^{\mathrm{iv}}-\mathrm{Al}-\mathrm{F} 5^{\mathrm{iii}}$ & $90.11(11)$ \\
\hline $\mathrm{F} 5^{\mathrm{iv}}-\mathrm{Ba}-\mathrm{F} 1^{\mathrm{v}}$ & $53.36(6)$ & $\mathrm{F} 2^{\mathrm{iv}}-\mathrm{Al}-\mathrm{F} 5^{\mathrm{iii}}$ & $175.02(10)$ \\
\hline $\mathrm{F} 3^{\mathrm{iv}}-\mathrm{Ba}-\mathrm{F} 1^{\mathrm{v}}$ & $50.92(7)$ & $\mathrm{F} 4^{\mathrm{xii}}-\mathrm{Al}-\mathrm{F} 3^{\mathrm{iii}}$ & $88.87(11)$ \\
\hline $\mathrm{F} 7-\mathrm{Ba}-\mathrm{F} 3$ & $59.02(6)$ & $\mathrm{F} 1-\mathrm{Al}-\mathrm{F} 3^{\mathrm{iii}}$ & $86.45(11)$ \\
\hline $\mathrm{F} 4 \mathrm{i}-\mathrm{Ba}-\mathrm{F} 3$ & $90.21(6)$ & $\mathrm{F} 6^{\mathrm{iv}}-\mathrm{Al}-\mathrm{F} 3^{\mathrm{iii}}$ & $175.99(12)$ \\
\hline $\mathrm{F} 1 \mathrm{i}-\mathrm{Ba}-\mathrm{F} 3$ & $111.81(7)$ & $\mathrm{F} 2^{\mathrm{iv}}-\mathrm{Al}-\mathrm{F} 3^{\mathrm{iii}}$ & $87.98(10)$ \\
\hline $\mathrm{F} 2 \mathrm{ii}-\mathrm{Ba}-\mathrm{F} 3$ & $52.19(6)$ & $\mathrm{F} 5^{\mathrm{iii}}-\mathrm{Al}-\mathrm{F} 3^{\mathrm{iii}}$ & $87.19(10)$ \\
\hline 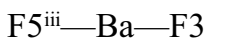 & $153.12(6)$ & $\mathrm{F} 7^{\mathrm{i}}-\mathrm{Fe}-\mathrm{F} 2^{\text {viii }}$ & $85.92(8)$ \\
\hline $\mathrm{F} 5^{\mathrm{iv}}-\mathrm{Ba}-\mathrm{F} 3$ & $113.88(6)$ & $\mathrm{F} 7^{\mathrm{x}}-\mathrm{Fe}-\mathrm{F} 2^{\text {viii }}$ & $94.08(8)$ \\
\hline $\mathrm{F} 3^{\mathrm{iv}}-\mathrm{Ba}-\mathrm{F} 3$ & $78.06(7)$ & $\mathrm{F} 7^{\mathrm{i}}-\mathrm{Fe}-\mathrm{F} 2^{\mathrm{xi}}$ & $94.08(8)$ \\
\hline $\mathrm{F} 1^{\mathrm{v}}-\mathrm{Ba}-\mathrm{F} 3$ & $61.81(6)$ & $\mathrm{F} 7^{\mathrm{x}}-\mathrm{Fe}-\mathrm{F} 2^{\mathrm{xi}}$ & $85.92(8)$ \\
\hline $\mathrm{F} 7-\mathrm{Ba}-\mathrm{F} 6^{\mathrm{iii}}$ & $149.97(6)$ & $\mathrm{F} 7^{\mathrm{i}}-\mathrm{Fe}-\mathrm{F} 3^{\mathrm{x}}$ & $97.13(8)$ \\
\hline $\mathrm{F} 4^{\mathrm{i}}-\mathrm{Ba}-\mathrm{F} 6^{\mathrm{iii}}$ & $127.50(6)$ & $\mathrm{F} 7^{\mathrm{x}}-\mathrm{Fe}-\mathrm{F} 3^{\mathrm{x}}$ & $82.87(8)$ \\
\hline $\mathrm{F} 1^{\mathrm{i}}-\mathrm{Ba}-\mathrm{F} 6^{\mathrm{iii}}$ & $50.94(6)$ & $\mathrm{F} 2^{\mathrm{viii}}-\mathrm{Fe}-\mathrm{F}^{\mathrm{x}}$ & $84.30(8)$ \\
\hline 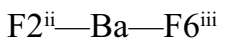 & $120.42(6)$ & $\mathrm{F} 2^{\mathrm{xi}}-\mathrm{Fe}-\mathrm{F} 3^{\mathrm{x}}$ & $95.70(8)$ \\
\hline 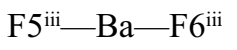 & $68.77(5)$ & $\mathrm{F} 7^{\mathrm{i}}-\mathrm{Fe}-\mathrm{F} 3^{\mathrm{i}}$ & $82.87(8)$ \\
\hline $\mathrm{F} 5^{\mathrm{iv}}-\mathrm{Ba}-\mathrm{F} 6^{\mathrm{iii}}$ & $56.89(6)$ & $\mathrm{F} 7^{\mathrm{x}}-\mathrm{Fe}-\mathrm{F} 3^{\mathrm{i}}$ & $97.13(8)$ \\
\hline $\mathrm{F} 3^{\mathrm{iv}}-\mathrm{Ba}-\mathrm{F} 6^{\mathrm{iii}}$ & $63.59(6)$ & $\mathrm{F} 2^{\mathrm{viii}}-\mathrm{Fe}-\mathrm{F} 3^{\mathrm{i}}$ & $95.70(8)$ \\
\hline $\mathrm{F} 1^{\mathrm{v}}-\mathrm{Ba}-\mathrm{F} 6^{\mathrm{iii}}$ & $103.02(6)$ & $\mathrm{F} 2^{\mathrm{xi}}-\mathrm{Fe}-\mathrm{F} 3^{\mathrm{i}}$ & $84.30(8)$ \\
\hline $\mathrm{F} 3-\mathrm{Ba}-\mathrm{F} 6^{\mathrm{iii}}$ & $137.04(6)$ & $\mathrm{Al}-\mathrm{F} 1-\mathrm{Ba}^{\mathrm{v}}$ & $114.10(11)$ \\
\hline $\mathrm{F} 7-\mathrm{Ba}-\mathrm{F} 6^{\mathrm{iv}}$ & $58.77(6)$ & $\mathrm{Al}-\mathrm{F} 1-\mathrm{Ba}^{\mathrm{i}}$ & $96.30(9)$ \\
\hline $\mathrm{F} 4^{\mathrm{i}}-\mathrm{Ba}-\mathrm{F} 6^{\mathrm{iv}}$ & $67.12(6)$ & $\mathrm{Ba}^{\mathrm{v}}-\mathrm{F} 1-\mathrm{Ba}^{\mathrm{i}}$ & $138.03(10)$ \\
\hline
\end{tabular}




\begin{tabular}{|c|c|}
\hline $\mathrm{F} 1^{\mathrm{i}}-\mathrm{Ba}-\mathrm{F} 6^{\mathrm{iv}}$ & $127.71(7)$ \\
\hline $\mathrm{F} 2^{\mathrm{ii}}-\mathrm{Ba}-\mathrm{F} 6^{\mathrm{iv}}$ & $130.72(6)$ \\
\hline $\mathrm{F} 5^{\mathrm{iii}}-\mathrm{Ba}-\mathrm{F}^{\mathrm{iv}}$ & $50.93(5)$ \\
\hline $\mathrm{F} 5^{\mathrm{iv}}-\mathrm{Ba}-\mathrm{F} 6^{\mathrm{iv}}$ & $67.24(5)$ \\
\hline $\mathrm{F} 3^{\mathrm{iv}}-\mathrm{Ba}-\mathrm{F} 6^{\mathrm{iv}}$ & $118.00(6)$ \\
\hline $\mathrm{F} 1^{\mathrm{v}}-\mathrm{Ba}-\mathrm{F} 6^{\mathrm{iv}}$ & $82.69(7)$ \\
\hline $\mathrm{F} 3-\mathrm{Ba}-\mathrm{F} 6^{\mathrm{iv}}$ & $117.62(5)$ \\
\hline $\mathrm{F} 6^{\mathrm{iii}}-\mathrm{Ba}-\mathrm{F} 6^{\mathrm{iv}}$ & $97.89(5)$ \\
\hline $\mathrm{F} 7-\mathrm{Ba}-\mathrm{F} 1^{\mathrm{vi}}$ & $105.96(6)$ \\
\hline $\mathrm{F} 4-\mathrm{Ba}-\mathrm{F} 1^{\mathrm{vi}}$ & $113.22(7)$ \\
\hline $\mathrm{F} 1^{\mathrm{i}}-\mathrm{Ba}-\mathrm{F} 1^{\mathrm{vi}}$ & $68.57(8)$ \\
\hline 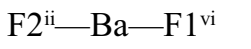 & $48.36(6)$ \\
\hline 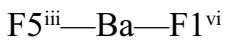 & $144.89(5)$ \\
\hline $\mathrm{F} 5^{\mathrm{iv}}-\mathrm{Ba}-\mathrm{F} 1^{\mathrm{vi}}$ & $111.48(6)$ \\
\hline $\mathrm{F} 3^{\mathrm{iv}}-\mathrm{Ba}-\mathrm{F} 1^{\mathrm{vi}}$ & $58.64(6)$ \\
\hline $\mathrm{F} 1^{\mathrm{v}}-\mathrm{Ba}-\mathrm{F} 1^{\mathrm{vi}}$ & $84.02(7)$ \\
\hline $\mathrm{F} 3-\mathrm{Ba}-\mathrm{F} 1^{\mathrm{vi}}$ & $46.95(6)$ \\
\hline $\mathrm{F} 6^{\mathrm{iii}-}-\mathrm{Ba}-\mathrm{F} 1^{\mathrm{vi}}$ & $94.27(6)$ \\
\hline $\mathrm{F} 6^{\mathrm{iv}}-\mathrm{Ba}-\mathrm{F} 1^{\mathrm{vi}}$ & $163.69(5)$ \\
\hline $\mathrm{F}^{\mathrm{vii}}-\mathrm{Ca}-\mathrm{F}^{\mathrm{i}}$ & $145.64(12)$ \\
\hline $\mathrm{F}^{\mathrm{vii}}-\mathrm{Ca}-\mathrm{F}^{\mathrm{iii}}$ & $80.29(8)$ \\
\hline $\mathrm{F} 7^{\mathrm{i}}-\mathrm{Ca}-\mathrm{F}^{\mathrm{iii}}$ & $78.04(8)$ \\
\hline $\mathrm{F} 7^{\mathrm{vii}}-\mathrm{Ca}-\mathrm{F}^{\mathrm{viii}}$ & $78.04(8)$ \\
\hline $\mathrm{F}^{\mathrm{i}}-\mathrm{Ca}-\mathrm{F}^{\text {viii }}$ & $80.29(8)$ \\
\hline $\mathrm{F}{ }^{\mathrm{iii}}-\mathrm{Ca}-\mathrm{F}^{\mathrm{viii}}$ & $100.95(11)$ \\
\hline $\mathrm{F}^{\mathrm{vii}}-\mathrm{Ca}-\mathrm{F} 4$ & $139.26(9)$ \\
\hline $\mathrm{F}^{\mathrm{i}}-\mathrm{Ca}-\mathrm{F} 4$ & $73.57(8)$ \\
\hline $\mathrm{F} 6^{\mathrm{iii}}-\mathrm{Ca}-\mathrm{F} 4$ & $133.25(7)$ \\
\hline $\mathrm{F}^{\mathrm{viii}}-\mathrm{Ca}-\mathrm{F} 4$ & $109.75(8)$ \\
\hline $\mathrm{F}^{\text {vii }}-\mathrm{Ca}-\mathrm{F} 4^{\mathrm{ix}}$ & $73.57(8)$ \\
\hline
\end{tabular}

\begin{tabular}{|c|c|}
\hline $\mathrm{Al}-\mathrm{F} 1-\mathrm{Ba}^{\mathrm{iii}}$ & $90.11(11)$ \\
\hline $\mathrm{Ba}^{\mathrm{v}}-\mathrm{F} 1-\mathrm{Ba}^{\mathrm{iii}}$ & $111.43(8)$ \\
\hline $\mathrm{Ba}-\mathrm{F} 1-\mathrm{Ba}^{\mathrm{iii}}$ & $95.98(7)$ \\
\hline $\mathrm{Al}{ }^{\mathrm{iv}}-\mathrm{F} 2-\mathrm{Fe}^{\mathrm{xiii}}$ & $136.08(11)$ \\
\hline $\mathrm{Al}^{\mathrm{iv}}-\mathrm{F} 2-\mathrm{Ba}^{\mathrm{xiv}}$ & $106.08(9)$ \\
\hline $\mathrm{Fe}^{\mathrm{xiii}}-\mathrm{F} 2-\mathrm{Ba}^{\mathrm{xiv}}$ & $117.52(8)$ \\
\hline $\mathrm{Alv}-\mathrm{F} 3-\mathrm{Fe}^{\mathrm{v}}$ & $125.66(11)$ \\
\hline $\mathrm{Al}^{\mathrm{vi}}-\mathrm{F} 3-\mathrm{Ba}^{\mathrm{iv}}$ & $94.81(9)$ \\
\hline $\mathrm{Fe}^{\mathrm{v}}-\mathrm{F} 3-\mathrm{Ba}^{\mathrm{iv}}$ & $131.32(9)$ \\
\hline 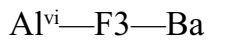 & $97.27(9)$ \\
\hline $\mathrm{Fe}-\mathrm{F} 3-\mathrm{Ba}$ & $98.64(7)$ \\
\hline $\mathrm{Ba}^{\mathrm{iv}}-\mathrm{F} 3-\mathrm{Ba}$ & $101.94(7)$ \\
\hline $\mathrm{Al}^{\mathrm{xv}}-\mathrm{F} 4-\mathrm{Ca}$ & $107.92(10)$ \\
\hline $\mathrm{Al}^{\mathrm{xv}}-\mathrm{F} 4-\mathrm{Ba}^{\mathrm{v}}$ & $142.62(11)$ \\
\hline $\mathrm{Ca}-\mathrm{F} 4-\mathrm{Ba}^{\mathrm{v}}$ & $109.17(8)$ \\
\hline $\mathrm{Al}^{\mathrm{vi}}-\mathrm{F} 5-\mathrm{Ca}^{\mathrm{iv}}$ & $99.48(9)$ \\
\hline $\mathrm{Al}^{\mathrm{vi}}-\mathrm{F} 5-\mathrm{Ba}^{\mathrm{vi}}$ & $116.53(9)$ \\
\hline $\mathrm{Ca}^{\mathrm{iv}}-\mathrm{F} 5-\mathrm{Ba}^{\mathrm{vi}}$ & $103.28(7)$ \\
\hline $\mathrm{Al}^{\mathrm{vi}}-\mathrm{F} 5-\mathrm{Ba}^{\mathrm{iv}}$ & $96.94(8)$ \\
\hline $\mathrm{Ca}^{\mathrm{iv}}-\mathrm{F} 5-\mathrm{Ba}^{\mathrm{iv}}$ & $117.45(7)$ \\
\hline $\mathrm{Ba}^{\mathrm{vi}}-\mathrm{F} 5-\mathrm{Ba}^{\mathrm{iv}}$ & $121.51(7)$ \\
\hline $\mathrm{Al}^{\mathrm{iv}}-\mathrm{F} 6-\mathrm{Ca}^{\mathrm{viii}}$ & $133.22(12)$ \\
\hline $\mathrm{Al}^{\mathrm{iv}}-\mathrm{F} 6-\mathrm{Ba}^{\mathrm{vi}}$ & $100.10(9)$ \\
\hline $\mathrm{Ca}^{\mathrm{viii}}-\mathrm{F} 6-\mathrm{Ba}^{\mathrm{vi}}$ & $113.52(8)$ \\
\hline $\mathrm{Al}^{\mathrm{iv}}-\mathrm{F} 6-\mathrm{Ba}^{\mathrm{iv}}$ & $102.40(9)$ \\
\hline $\mathrm{Ca}^{\text {viii }}-\mathrm{F} 6-\mathrm{Ba}^{\mathrm{iv}}$ & $101.01(7)$ \\
\hline $\mathrm{Ba}^{\mathrm{vi}}-\mathrm{F} 6-\mathrm{Ba}^{\mathrm{iv}}$ & $102.45(6)$ \\
\hline $\mathrm{Fe}^{\mathrm{v}}-\mathrm{F} 7-\mathrm{Ca}^{\mathrm{xvi}}$ & $120.99(11)$ \\
\hline $\mathrm{Fe}^{\mathrm{v}}-\mathrm{F} 7-\mathrm{Ba}$ & $113.91(9)$ \\
\hline $\mathrm{Ca}^{\mathrm{xvi}}-\mathrm{F} 7-\mathrm{Ba}$ & $120.85(10)$ \\
\hline
\end{tabular}

Symmetry codes: (i) $-x+1 / 2, y+1 / 2,-z+1 / 2$; (ii) $-x+1 / 2, y+1 / 2,-z+3 / 2$; (iii) $x,-y+1, z-1 / 2$; (iv) $-x+1 / 2,-y+1 / 2,-z+1$; (v) $-x+1 / 2, y-1 / 2,-z+1 / 2$; (vi) $x$, $-y+1, z+1 / 2$; (vii) $x-1 / 2, y+1 / 2, z$; (viii) $-x,-y+1,-z+1$; (ix) $-x, y,-z+1 / 2$; (x) $x-1 / 2,-y+1 / 2, z-1 / 2$; (xi) $x, y, z-1$; (xii) $x+1 / 2, y+1 / 2, z$; (xiii) $x, y, z+1$; (xiv) $-x+1 / 2, y-1 / 2,-z+3 / 2$; (xv) $x-1 / 2, y-1 / 2, z$; (xvi) $x+1 / 2, y-1 / 2, z$. 